\title{
Hydrochemical characteristics and element contents of natural waters in Tibet, China
}

\author{
TIAN Yuan ${ }^{1,2},{ }^{*}$ YU Chengqun ${ }^{1},{ }^{*}$ LUO Kunli ${ }^{1}$, ZHA Xinjie $^{3}$, WU Jianshuang ${ }^{1}$, \\ ZHANG Xianzhou ${ }^{1}$, NI Runxiang ${ }^{1,2}$ \\ 1. Institute of Geographic Sciences and Natural Resources Research, CAS, Beijing 100101, China; \\ 2. University of Chinese Academy of Sciences, Beijing 100049, China; \\ 3. Xi'an University of Science and Technology, Xi'an 710054, China
}

\begin{abstract}
Sixty water samples (35 groundwater samples, 22 surface water samples and three hot-spring water samples) were collected at 36 points from villages and towns in Lhasa city, Nagchu (Nagqu) prefecture, Ali (Ngari) prefecture and Shigatse (Xigaze) prefecture (Tibet) in 2013 to study the hydrochemical characteristics and element contents of natural waters. The concentrations of elements were determined in the water samples and compared with the concentrations in water samples from other regions, such as southeast Qinghai, south Xinjiang, east Sichuan and west Tibet. The hydrochemical species in different areas were also studied. Water in most parts of Tibet reaches the requirements of the Chinese national standard and the World Health Organization international standard. The $\mathrm{pH}$ values of the water samples ranged from 6.75 to 8.21 and the value for the mean total dissolved solids was $225.54 \mathrm{mg} / \mathrm{L}$. The concentration of arsenic in water from Ali prefecture exceeded the limit of both the Chinese national standard and the international standard and the concentration of fluoride in water from Shuanghu exceeded the limit of both the Chinese national standard and the international standard. The main hydrochemical species in water of Tibet is $\mathrm{Ca}\left(\mathrm{HCO}_{3}\right)_{2}$. From south to north, the main cation in water changes from $\mathrm{Ca}^{2+}$ to $\mathrm{Na}^{+}$, whereas the main anions in water change from $\mathrm{HCO}_{3}{ }^{-}$to $\mathrm{Cl}^{-}$and $\mathrm{SO}_{4}{ }^{2-}$. The chemistry of river water and melt water from ice and snow is dominated by the rocks present at their source, whereas the chemistry of groundwater is affected by many factors. Tectonic divisions determine the concentrations of the main elements in water and also affect the hydrochemical species present.
\end{abstract}

Keywords: element concentrations; hydrochemical species; water chemistry; water quality; Tibet

\section{Introduction}

The Tibet Autonomous Region $\left(26^{\circ} 44^{\prime}-36^{\circ} 32^{\prime} \mathrm{N}, 78^{\circ} 25^{\prime}-99^{\circ} 06^{\prime} \mathrm{E}\right)$ is located on the Qing-

Received: 2014-10-20 Accepted: 2014-11-16

Foundation: National Key Technologies R\&D Program in the 12th Five-Year Plan of China, No.2011BAD17B05-4, No. 2011BAC09B03; National Key Basic Research Program of China (973 Program), No.2014CB238906; National Natural Science Foundation of China, No.40872210, No.41172310, No.40171006

Author: Tian Yuan (1991-), Graduate student in Institute of Geographic Sciences and Natural Resources Research, CAS, specialized in geology and health, environmental science. E-mail: tiany.13s@igsnrr.ac.cn

"Corresponding author: Yu Chengqun, Professor, E-mail: yucq@igsnrr.ac.cn Luo Kunli, Professor, E-mail: luokl@igsnrr.ac.cn 
hai-Tibet Plateau on the southwestern border of China. It has a land area of $1.22 \times 10^{6} \mathrm{~km}^{2}$ with an average elevation being $>4 \mathrm{~km}$ above sea level. The geology of Tibet is complicated and diverse and there are unique geological hazards in this region (Shen et al., 2011).

According to China's Water Resource Report in 2007 (MWR, 2009), the inflowing rivers and lake water in Tibet have enormous capacity, low elements concentrations and are of good quality; the waters are mainly composed of rain, ice/snow melt water and groundwater. The average volume of surface water resources in Tibet is $4.394 \times 10^{11} \mathrm{~m}^{3}$ per year, which accounts for $17 \%$ of the total surface water resources of China's mainland. The total volume of groundwater resources in Tibet is about $9.661 \times 10^{10} \mathrm{~m}^{3}$. The cover area and volume of the glaciers in Tibet account for $48.2 \%$ of the surface cover and $53.6 \%$ of the total volume of the glaciers in China (MWR, 2009; Bian et al., 2010). The volume of ice/snow melt water in Tibet is about $3 \times 10^{11} \mathrm{~m}^{3}$.

Zhang et al. (2013) analyzed the quality of drinking water in Nyingchi, Tibet in 2011, which showed that the water quality in Nyingchi did not often reach the required standard for drinking water. Liu and Ge (2012) determined trace elements in lake water from a mining area using the inductively coupled plasma mass spectrometry (ICP-MS) and compared the results with those obtained by four other methods to determine the optimum sample recovery rate. $\mathrm{Bu}$ et al. (2011) determined the concentration of arsenic in three fish ponds in Lhasa. The arsenic concentration in Quxu Niedang fish pond was the highest at $>60 \mu \mathrm{g} / \mathrm{L}$. Nie et al. (2011) analyzed the microbiological indicators in drinking water from counties in Lhasa and found that the water quality did not often reach the required microbiological standards for drinking water. Wang et al. (2013) studied the hydrochemical characteristics of Lake Manasarovar and Lake Rakshastal and showed that the $\mathrm{pH}$ was inversely proportional to the amount of dissolved oxygen. Zheng et al. (2007a, 2007b, 2008) monitored and tested the water quality in some areas of Naqu and Biru where troops were stationed; the water samples did not often reach the required standard for drinking water and the arsenic concentration commonly exceeded the Chinese national standard. Zhang et al. (2009) and Li et al. (2010) analyzed the hydrochemical characteristics of water samples collected from the Niyang and Yarlung Zangbo rivers in Tibet and reported some basic data for the water samples. Liu et al. (2013) analyzed the results of a drinking water safety project in rural areas of Shannan in 2012; the results showed that some water samples were polluted. Luo et al. (2010) surveyed the water quality of about 140 urban drinking water sources in Tibet and found that $96.9 \%$ of the drinking water samples in urban areas met the required standard for drinking water. Zhao et al. (2002) investigated the water quality of self-supplied water sources in some remote areas where troops were stationed and water sanitation was poor.

There have been few systematic studies of the geochemical features and element concentrations of waters in Tibet. We investigated the elemental composition and distribution of water in Tibet in August 2013 (Figure 1) and collected water samples for analysis. From September to October, we determined the elemental composition and hydrochemical characteristics of water samples at the Institute of Geographic Sciences and Natural Resources Research, Chinese Academy of Sciences (IGSNRR, CAS). We also studied the hydrochemical and element characteristics of these water samples. This paper is a comprehensive report of the concentration and distribution of elements in waters from different geological areas of Tibet. 


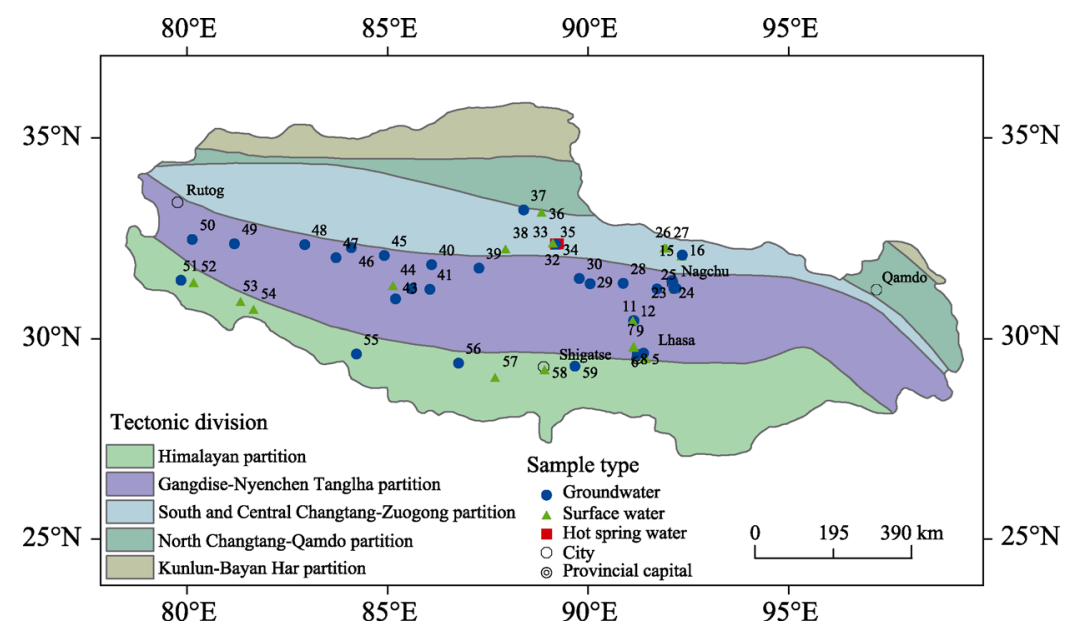

Figure 1 Geographical location, tectonic divisions (based on Ma et al., 2002) and distribution of water sampling points in Tibet

\section{Study areas and methods}

\subsection{Sampling sites and types of samples}

Sixty water samples (35 groundwater samples, 22 surface water samples and three hot-spring samples) were collected (Tables 1 and 2) from the majority of counties in Lhasa

Table 1 Distribution of surface water sampling locations in Tibet

\begin{tabular}{|c|c|c|c|c|c|c|}
\hline $\begin{array}{c}\text { Sample } \\
\text { No. }\end{array}$ & Date & $\begin{array}{c}\text { Latitude } \\
\left({ }^{\circ}\right)\end{array}$ & $\begin{array}{c}\text { Longitude } \\
\left({ }^{\circ}\right)\end{array}$ & $\begin{array}{l}\text { Eleva- } \\
\text { tion }(\mathrm{m})\end{array}$ & Sample site & Sample type \\
\hline 1 & 2013.04 .27 & 29.566389 & 91.401144 & 4196 & No.7 team, Xincang village, Dazi county & River water \\
\hline 2 & 2013.04 .27 & 29.570000 & 91.393056 & 4287 & No.7 team, Xincang village, Dazi county & Ice/snow melt \\
\hline 5 & 2013.07 .13 & 29.604167 & 91.380833 & 3970 & No.6 team, Xincang village, Dazi county & River water \\
\hline 6 & 2013.07.20 & 29.873056 & 91.093333 & 3972 & Bailang village, Linzhou county & River water \\
\hline 7 & 2013.07.20 & 29.873056 & 91.093333 & 3972 & Bailang village, Linzhou county & River water \\
\hline 8 & 2013.07.20 & 29.871667 & 91.092500 & 3954 & Bailang village, Linzhou county & River water \\
\hline 9 & 2013.07 .20 & 29.850000 & 91.092222 & 4041 & Bailang village, Linzhou county & River water \\
\hline 12 & 2013.07.24 & 30.495000 & 91.069167 & 4317 & Dangxiong field station & Ice/snow melt \\
\hline 14 & 2013.08 .04 & 32.105833 & 92.285278 & 4645 & Nyainrong, Nyainrong county & River water \\
\hline 15 & 2013.08 .04 & 32.109722 & 92.302500 & 4634 & Nyainrong, Nyainrong county & Ice/snow melt \\
\hline 21 & 2013.08 .05 & 31.273333 & 92.102778 & 4450 & Kema village, Luoma town, Nagchu & River water \\
\hline 26 & 2013.08 .08 & 32.301111 & 91.868056 & 4683 & Amdo county & River water \\
\hline 27 & 2013.08 .08 & 32.303333 & 91.906944 & 4732 & Shenkagang village, Amdo county & River water \\
\hline 35 & 2013.08 .11 & 32.433056 & 89.081111 & 4791 & Duoma township, Shuanghu county & River water \\
\hline 36 & 2013.08 .11 & 33.206944 & 88.798611 & 4981 & Shuanghu county & Ice/snow melt \\
\hline 38 & 2013.08 .12 & 32.286944 & 87.901944 & 4754 & Cuozheluoma town, Shuanghu county & River water \\
\hline 44 & 2013.08 .17 & 31.370000 & 85.091667 & 4728 & Gerze county & River water \\
\hline 52 & 2013.08 .21 & 31.451111 & 80.120556 & 4675 & Alzadar county & River water \\
\hline 53 & 2013.08 .21 & 30.970278 & 81.285000 & 4679 & Baga town, Burang county & Ice/snow melt \\
\hline 54 & 2013.08 .22 & 30.776389 & 81.613611 & 4625 & Huoer town, Burang county & Ice/snow melt \\
\hline 57 & 2013.08 .22 & 29.088611 & 87.637778 & 4048 & Lhatse county & River water \\
\hline 58 & 2013.18 .23 & 29.278889 & 88.879167 & 3777 & Shigatse & River water \\
\hline
\end{tabular}


Table 2 Distribution of groundwater sampling locations in Tibet

\begin{tabular}{|c|c|c|c|c|c|c|}
\hline $\begin{array}{l}\text { Sample } \\
\text { number }\end{array}$ & Date & Latitude $\left(^{\circ}\right)$ & $\begin{array}{l}\text { Longitude } \\
\left({ }^{\circ}\right)\end{array}$ & $\begin{array}{l}\text { Eleva- } \\
\text { tion (m) }\end{array}$ & Sample site & $\begin{array}{l}\text { Depth } \\
\text { (m) }\end{array}$ \\
\hline 3 & 2013.04 .28 & 29.642406 & 91.180731 & 3666 & Tibet University, Lhasa & - \\
\hline 4 & 2013.04 .28 & 29.642950 & 91.180108 & 3666 & Tibet University, Lhasa & 6 \\
\hline 10 & 2013.07 .23 & 30.476389 & 91.099722 & 4297 & Damxung county & 5 \\
\hline 11 & 2013.07 .23 & 30.477500 & 91.103056 & 4281 & Damxung county & 30 \\
\hline 13 & 2013.08 .03 & 31.475908 & 92.062089 & 4521 & Nagchu town, Nagqu county & - \\
\hline 16 & 2013.08 .04 & 32.110000 & 92.303611 & 4612 & Nyainrong & 10 \\
\hline 17 & 2013.08 .04 & 31.465556 & 92.062778 & 4508 & Nagchu town, Nagchu & 10 \\
\hline 18 & 2013.08 .05 & 31.468611 & 92.059167 & 4534 & Nagchu town, Nagchu & - \\
\hline 19 & 2013.08 .05 & 31.271944 & 92.158611 & 4471 & Kema village, Luoma town, Nagqu county & 10 \\
\hline 20 & 2013.08 .05 & 31.271944 & 92.160000 & 4439 & Kema village, Luoma town, Nagqu county & 5 \\
\hline 22 & 2013.08 .05 & 31.469167 & 92.047500 & 4523 & Nagchu town, Nagqu county & 27 \\
\hline 23 & 2013.08 .05 & 31.275833 & 92.105278 & 4459 & Nagqu field station, Nagqu county & 10 \\
\hline 24 & 2013.08 .06 & 31.471944 & 92.047500 & 4506 & Nagqu Agriculture Bureau, Nagqu county & - \\
\hline 25 & 2013.08 .07 & 32.264050 & 91.681160 & 4685 & Amdo county & - \\
\hline 28 & 2013.08 .09 & 31.405278 & 90.835278 & 4606 & Baila town, Baingoin county & 6 \\
\hline 29 & 2013.08 .09 & 31.395000 & 90.007778 & 4703 & Baingoin county & 15 \\
\hline 30 & 2013.08 .10 & 31.523333 & 89.741111 & 4576 & Mendang town, Baingoin county & 4 \\
\hline 31 & 2013.08 .10 & 32.390000 & 89.218889 & 4703 & Duoma town, Shuanghu county $\left(40^{\circ} \mathrm{C}\right)$ & - \\
\hline 32 & 2013.08 .10 & 32.386667 & 89.149167 & 4701 & Duoma township, Shuanghu county $\left(50^{\circ} \mathrm{C}\right)$ & - \\
\hline 33 & 2013.08 .10 & 32.388056 & 89.149722 & 4708 & Duoma township, Shuanghu county $\left(60^{\circ} \mathrm{C}\right)$ & - \\
\hline 34 & 2013.08 .10 & 32.384444 & 89.145278 & 4705 & Duoma town, Shuanghu county & 15 \\
\hline 37 & 2013.08 .12 & 33.233056 & 88.352222 & 4811 & Jiacuo, Shuanghu county & 4 \\
\hline 39 & 2013.08 .13 & 31.786111 & 87.234722 & 4553 & Nyima county & - \\
\hline 40 & 2013.08 .15 & 31.876944 & 86.061667 & 4797 & Asuo town, Nyima county & 6 \\
\hline 41 & 2013.08 .15 & 31.253056 & 86.014722 & 4726 & Juncang town, Nyima county & 8 \\
\hline 42 & 2013.08 .16 & 31.263333 & 85.563056 & 4690 & Cishi town, Cuoqin county & 10 \\
\hline 43 & 2013.08 .16 & 31.018056 & 85.153889 & 4649 & Cuoqin county & 10 \\
\hline 45 & 2013.08 .17 & 32.098611 & 84.878889 & 4433 & Dongco, Gerze county & - \\
\hline 46 & 2013.08 .17 & 32.300000 & 84.055278 & 4447 & Gerze county & 11 \\
\hline 47 & 2013.08 .18 & 32.040697 & 83.669022 & 4398 & Marm, Gerze county & 5 \\
\hline 48 & 2013.08 .19 & 32.371667 & 82.891111 & 4474 & Wenbudangsang town, Geji county & - \\
\hline 49 & 2013.08 .19 & 32.388333 & 81.143333 & 4542 & Geji county & 5 \\
\hline 50 & 2013.08 .20 & 32.503333 & 80.090000 & 4308 & Seng-ge Kambab & - \\
\hline 51 & 2013.08 .21 & 31.481389 & 79.801111 & 3755 & Tholing, Zanda county & 35 \\
\hline 55 & 2013.08 .22 & 29.652169 & 84.181464 & 4602 & Laozhongba town, Zhongba county & 6 \\
\hline 56 & 2013.08 .22 & 29.419006 & 86.724014 & 4632 & Sangsang town, Ngamring county & 10 \\
\hline 59 & 2013.18 .23 & 29.346667 & 89.635833 & 3752 & Dazhu village, Shigatse & 15 \\
\hline 60 & 2013.08 .24 & 29.676389 & 91.344722 & 3699 & Dazi county Lhasa field station & 30 \\
\hline
\end{tabular}


city, Nagchu prefecture, Ali prefecture and Shigatse prefecture in Tibet. The tap water for everyday use in Tibet comes from untreated shallow wells, river water and ice/snow melt water. These samples (nine samples from the Himalayan partition, 38 samples from Gangdise-Nyenchen Tanglha partition and 13 samples from the South and Central Changtang-Zuogong partition) therefore reflected the range of hydrochemical characteristics and types of natural water in Tibet.

\subsection{Sampling and analysis}

\subsubsection{Sampling and preservation}

The authors strictly complied with the methods in Monitoring and Analysis Methods for Water and Waste Water (MEP, 2002) to collect water samples from different geological areas in Tibet in August 2013. The sample containers used were colorless $0.5 \mathrm{~L}$ polythene plastic barrels and were dipped into nitric acid for $24 \mathrm{~h}$ before use. The containers were then washed sequentially with $10 \%$ hydrochloric acid solution and tap water. The containers were then washed with distilled water and finally flushed three times with sampling water.

\subsubsection{Methods of analysis}

The water samples were analyzed at the IGSNRR, CAS. The $\mathrm{pH}, E_{\mathrm{c}}$, RES, salinity (SAL), total dissolved solids (TDS) and temperature were determined using a Switzerland Mettler Toledo $\mathrm{pH}$ tester (SevenGo SG2) and Switzerland Mettler Toledo $E_{\mathrm{c}}$ tester (SevenGo SG3). The $E_{\mathrm{h}}$ was determined in situ using a Shanghai Sanxin ORP tester (SX712). The total hardness $(\mathrm{TH})$ was calculated from the concentrations of $\mathrm{Ca}^{2+}$ and $\mathrm{Mg}^{2+}$. The alkalinity (as $\mathrm{HCO}_{3}{ }^{-}$) was determined by acid-base titration (MH, 1985; MEP, 2002). The chloride $\left(\mathrm{Cl}^{-}\right)$ concentration of the water samples was determined using a chloride ion-selective electrode (Hirokazu et al., 1985; Yu et al., 2010) and the fluoride ( $\mathrm{F}^{-}$) concentration was determined using a fluoride ion-selective electrode (MH, 1985; MEP, 2002). All water samples were stored in pre-cleaned plastic bottles at $4{ }^{\circ} \mathrm{C}$ before analysis. Selenium and arsenic were determined by hydride generation atomic fluorescence spectrometry (MH, 1985; MEP, 2002). The concentrations of the major cations $\left(\mathrm{Ca}^{2+}, \mathrm{Mg}^{2+}, \mathrm{Na}^{+}, \mathrm{K}^{+}, \mathrm{P}, \mathrm{Sr}, \mathrm{B}\right.$ and $\left.\mathrm{SiO}_{2}\right)$ and the $\mathrm{SO}_{4}{ }^{2-}$ anion were determined by inductively coupled plasma atomic emission spectrometry (Optima 5300 DV, Waltham, Massachusetts in the United States, PerkinElmer). The concentrations of trace elements $(\mathrm{Li}, \mathrm{Zn}, \mathrm{U}, \mathrm{Rb}, \mathrm{Ba}$, $\mathrm{Bi}, \mathrm{Co}, \mathrm{Cs}, \mathrm{Ga}, \mathrm{In}, \mathrm{Ti}, \mathrm{V}, \mathrm{Ag}, \mathrm{Al}, \mathrm{Be}, \mathrm{Cd}, \mathrm{Cr}$, $\mathrm{Cu}, \mathrm{Fe}, \mathrm{Hg}, \mathrm{Mn}, \mathrm{Mo}, \mathrm{Ni}, \mathrm{Pb}$ and $\mathrm{Tl}$ ) were determined by ICP-MS (PerkinElmer, DRC-e). In each test method, a parallel sample was determined to ensure the stability of the data after every 20 samples. The percentage error in all the samples ranged from $\pm 0.19 \%$ to $\pm 4.96 \%$, i.e. less than $\pm 5 \%$ (Figure 2). Therefore these data are accurate and dependable

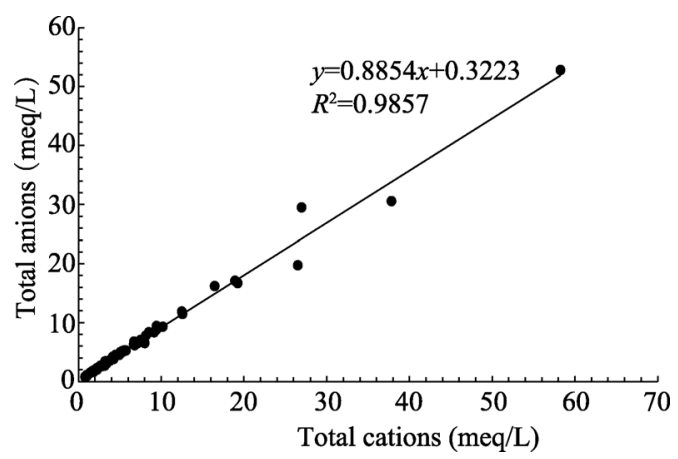

Figure 2 Total cations versus total anions in water samples from Tibet (Shen et al., 1993; Ji et al., 2007). 


\section{Results}

\subsection{Hydrochemical characteristics and element concentrations}

The water samples collected from Tibet have good hydrochemical and sensory characteristics (Tables 3 and 4). The $\mathrm{pH}$ in these samples ranged from 6.75 to 8.21 , with a mean value of 7.54. The average TDS was about $225.54 \mathrm{mg} / \mathrm{L}$, except for the hot-spring water. Because the majority of the water samples were collected from surface runoff, which is mainly recharged by ice/snow melt, the hardness of these samples ranged from soft to moderately hard.

\subsection{Trace element concentrations}

The background concentrations of elements from different geological settings not only affect the hydrochemical characteristics of the major elements in water, but also affect the hydrochemical characteristics of the trace elements (Warren, 1989; Webster, 1994). The

Table 3 Hydrochemical characteristics and element concentrations in surface water samples from Tibet

\begin{tabular}{|c|c|c|c|c|c|c|c|c|c|c|c|c|c|}
\hline $\begin{array}{l}\text { Sample } \\
\text { number }\end{array}$ & $\mathrm{pH}$ & $\begin{array}{c}E_{\mathrm{h}} \\
(\mathrm{mV})\end{array}$ & $\begin{array}{c}E_{c} \\
(\mu \mathrm{s} / \mathrm{cm})\end{array}$ & $\begin{array}{c}\text { TDS } \\
(\mathrm{mg} / \mathrm{L})\end{array}$ & $\begin{array}{c}\mathrm{TH} \\
(\mathrm{mg} / \mathrm{L})\end{array}$ & $\begin{array}{c}\mathrm{Ca}^{2+} \\
(\mathrm{mg} / \mathrm{L})\end{array}$ & $\begin{array}{c}\mathrm{Mg}^{2+} \\
(\mathrm{mg} / \mathrm{L})\end{array}$ & $\begin{array}{c}\mathrm{Na}^{+} \\
(\mathrm{mg} / \mathrm{L})\end{array}$ & $\begin{array}{c}\mathrm{K}^{+} \\
(\mathrm{mg} / \mathrm{L})\end{array}$ & $\begin{array}{c}\mathrm{HCO}_{3} \\
(\mathrm{mg} / \mathrm{L})\end{array}$ & $\begin{array}{c}\mathrm{SO}_{4}{ }^{2-} \\
(\mathrm{mg} / \mathrm{L})\end{array}$ & $\begin{array}{c}\mathrm{Cl}^{-} \\
(\mathrm{mg} / \mathrm{L})\end{array}$ & $\begin{array}{c}\mathrm{SiO}_{2} \\
(\mathrm{mg} / \mathrm{L})\end{array}$ \\
\hline 1 & 7.62 & 336 & 179 & 90 & 84 & 28.9 & 2.8 & 2.2 & 0.7 & 39.7 & 32.6 & 11.0 & 6.8 \\
\hline 2 & 7.30 & 293 & 197 & 99 & 105 & 40.2 & 1.2 & 1.9 & 0.3 & 91.5 & 10.6 & 6.1 & 10.2 \\
\hline 5 & 7.36 & 220 & 151 & 76 & 77 & 26.6 & 2.6 & 2.4 & 0.4 & 54.9 & 3.2 & 12.8 & 12.6 \\
\hline 6 & 7.45 & 224 & 96 & 48 & 44 & 13.6 & 2.4 & 2.7 & 0.7 & 15.3 & 12.0 & 8.7 & 6.5 \\
\hline 7 & 7.38 & 217 & 80 & 40 & 37 & 11.3 & 2.0 & 1.4 & 0.5 & 9.2 & 11.5 & 9.0 & 5.7 \\
\hline 8 & 7.29 & 235 & 95 & 47 & 44 & 13.6 & 2.4 & 2.8 & 0.7 & 21.4 & 12.2 & 10.1 & 6.6 \\
\hline 9 & 7.31 & 227 & 76 & 38 & 36 & 11.2 & 1.9 & 1.4 & 0.5 & 10.7 & 11.9 & 6.6 & 5.8 \\
\hline 12 & 7.15 & 226 & 133 & 66 & 68 & 21.5 & 3.5 & 1.3 & 0.8 & 39.7 & 21.5 & 8.8 & 4.5 \\
\hline 14 & 7.77 & 202 & 263 & 131 & 133 & 38.3 & 9.0 & 9.7 & 1.5 & 122.0 & 7.8 & 7.2 & 9.1 \\
\hline 15 & 7.55 & 209 & 348 & 174 & 186 & 53.0 & 12.8 & 9.7 & 1.2 & 155.6 & 12.6 & 34.3 & 10.4 \\
\hline 21 & 7.37 & 213 & 187 & 93 & 78 & 20.8 & 6.3 & 13.9 & 1.6 & 73.2 & 12.3 & 10.0 & 8.8 \\
\hline 26 & 7.65 & 185 & 529 & 265 & 268 & 67.1 & 24.1 & 27.1 & 3.0 & 268.5 & 59.8 & 14.4 & 7.6 \\
\hline 27 & 7.81 & 175 & 401 & 200 & 217 & 69.8 & 10.1 & 14.6 & 2.0 & 253.2 & 8.6 & 9.6 & 11.0 \\
\hline 35 & 7.80 & 185 & 1530 & 765 & 443 & 73.0 & 62.5 & 218.7 & 3.9 & 192.2 & 521.0 & 98.9 & 8.7 \\
\hline 36 & 8.02 & 172 & 344 & 172 & 180 & 26.3 & 27.5 & 11.9 & 3.7 & 125.1 & 32.8 & 27.5 & 9.1 \\
\hline 38 & 8.21 & 168 & 278 & 139 & 158 & 45.4 & 10.6 & 4.7 & 1.3 & 94.6 & 44.3 & 12.4 & 8.5 \\
\hline 44 & 7.90 & 188 & 368 & 184 & 196 & 58.6 & 11.8 & 11.8 & 1.8 & 155.6 & 56.3 & 13.6 & 11.8 \\
\hline 52 & 7.72 & 209 & 595 & 298 & 352 & 75.8 & 38.9 & 9.0 & 4.3 & 167.8 & 158.5 & 17.5 & 9.7 \\
\hline 53 & 7.90 & 200 & 172 & 86 & 89 & 23.8 & 7.0 & 4.4 & 0.7 & 18.3 & 39.3 & 21.9 & 4.7 \\
\hline 54 & 7.96 & 207 & 90 & 45 & 43 & 12.8 & 2.6 & 3.1 & 0.9 & 3.1 & 12.3 & 20.3 & 6.2 \\
\hline 57 & 7.64 & 219 & 226 & 113 & 121 & 40.3 & 4.9 & 4.6 & 0.8 & 79.3 & 30.7 & 15.4 & 9.4 \\
\hline 58 & 7.73 & 225 & 355 & 178 & 194 & 50.9 & 15.9 & 8.9 & 0.5 & 128.1 & 43.6 & 18.3 & 18.3 \\
\hline
\end{tabular}


Table 4 Hydrochemical characteristics and element contents in groundwater samples from Tibet

\begin{tabular}{|c|c|c|c|c|c|c|c|c|c|c|c|c|c|}
\hline $\begin{array}{l}\text { Sample } \\
\text { number }\end{array}$ & $\mathrm{pH}$ & $\begin{array}{c}E_{\mathrm{h}} \\
(\mathrm{mV})\end{array}$ & $\begin{array}{c}E_{c}(\mu \mathrm{s} / \\
\mathrm{cm})\end{array}$ & $\begin{array}{c}\text { TDS } \\
(\mathrm{mg} / \mathrm{L})\end{array}$ & $\begin{array}{c}\mathrm{TH} \\
(\mathrm{mg} / \mathrm{L})\end{array}$ & $\begin{array}{c}\mathrm{Ca}^{2+} \\
(\mathrm{mg} / \mathrm{L})\end{array}$ & $\begin{array}{c}\mathrm{Mg}^{2+} \\
(\mathrm{mg} / \mathrm{L})\end{array}$ & $\begin{array}{c}\mathrm{Na}^{+} \\
(\mathrm{mg} / \mathrm{L})\end{array}$ & $\begin{array}{c}\mathrm{K}^{+} \\
(\mathrm{mg} / \mathrm{L})\end{array}$ & $\begin{array}{l}\mathrm{HCO}_{3}^{-} \\
(\mathrm{mg} / \mathrm{L})\end{array}$ & $\begin{array}{c}\mathrm{SO}_{4}{ }^{2-} \\
(\mathrm{mg} / \mathrm{L})\end{array}$ & $\begin{array}{c}\mathrm{Cl}^{-} \\
(\mathrm{mg} / \mathrm{L})\end{array}$ & $\begin{array}{c}\mathrm{SiO}_{2} \\
(\mathrm{mg} / \mathrm{L})\end{array}$ \\
\hline 3 & 7.28 & 262 & 250 & 125 & 119 & 38.1 & 5.6 & 7.0 & 1.3 & 91.5 & 21.3 & 8.5 & 12.7 \\
\hline 4 & 7.31 & 244 & 261 & 131 & 124 & 38.6 & 6.5 & 7.4 & 1.5 & 85.4 & 27.2 & 9.5 & 9.6 \\
\hline 10 & 6.95 & 245 & 279 & 139 & 134 & 39.9 & 8.1 & 10.5 & 1.5 & 103.7 & 10.4 & 15.8 & 16.9 \\
\hline 11 & 7.11 & 233 & 161 & 81 & 83 & 24.3 & 5.3 & 3.2 & 1.0 & 64.1 & 6.4 & 10.0 & 11.1 \\
\hline 13 & 7.10 & 252 & 1587 & 793 & 605 & 131.4 & 66.5 & 152.2 & 7.8 & 485.1 & 156.0 & 182.1 & 10.0 \\
\hline 16 & 7.37 & 213 & 600 & 300 & 320 & 112.3 & 9.4 & 13.7 & 1.8 & 280.7 & 29.6 & 26.6 & 10.6 \\
\hline 17 & 7.28 & 230 & 1399 & 699 & 563 & 102.4 & 73.8 & 112.0 & 4.0 & 515.6 & 143.7 & 152.8 & 9.0 \\
\hline 18 & 7.81 & 205 & 404 & 202 & 165 & 31.7 & 20.6 & 33.7 & 3.7 & 155.6 & 44.1 & 16.3 & 6.8 \\
\hline 19 & 7.37 & 220 & 467 & 233 & 237 & 69.1 & 15.4 & 19.7 & 3.2 & 192.2 & 35.3 & 18.1 & 25.5 \\
\hline 20 & 6.93 & 235 & 458 & 229 & 227 & 61.9 & 17.4 & 18.6 & 4.1 & 167.8 & 46.0 & 16.2 & 33.1 \\
\hline 22 & 7.07 & 243 & 2480 & 124 & 667 & 151.4 & 69.2 & 292.0 & 13.6 & 167.1 & 95.5 & 860.6 & 12.6 \\
\hline 23 & 7.34 & 222 & 732 & 366 & 216 & 52.2 & 20.5 & 103.2 & 5.0 & 378.3 & 44.3 & 32.0 & 9.4 \\
\hline 24 & 7.20 & 220 & 1055 & 528 & 445 & 134.0 & 26.4 & 77.6 & 5.4 & 387.5 & 94.5 & 89.7 & 15.4 \\
\hline 25 & 7.37 & 207 & 554 & 277 & 282 & 69.2 & 26.1 & 23.3 & 2.9 & 259.3 & 49.5 & 17.2 & 9.6 \\
\hline 28 & 7.86 & 191 & 337 & 168 & 169 & 33.2 & 20.6 & 15.8 & 1.5 & 198.3 & 12.0 & 10.5 & 10.6 \\
\hline 29 & 7.56 & 210 & 804 & 402 & 389 & 126.2 & 17.5 & 34.5 & 3.6 & 405.8 & 52.7 & 15.6 & 15.4 \\
\hline 30 & 7.79 & 195 & 307 & 154 & 148 & 32.6 & 15.9 & 16.7 & 2.5 & 61.0 & 19.3 & 65.0 & 10.1 \\
\hline 31 & 6.75 & 245 & 1999 & 1000 & 472 & 144.4 & 26.7 & 363.3 & 20.8 & 970.2 & 86.4 & 35.3 & 25.9 \\
\hline 32 & 7.46 & 226 & 4360 & 2180 & 182 & 48.7 & 14.5 & 1171.0 & 53.4 & 2785.6 & 196.3 & 57.5 & 31.5 \\
\hline 33 & 7.19 & 220 & 2970 & 1487 & 183 & 57.3 & 9.5 & 732.3 & 33.8 & 1424.8 & 137.8 & 40.1 & 89.9 \\
\hline 34 & 7.55 & 180 & 972 & 486 & 315 & 81.6 & 26.5 & 131.5 & 8.5 & 585.8 & 43.0 & 18.1 & 22.9 \\
\hline 37 & 7.77 & 189 & 693 & 346 & 336 & 79.6 & 32.8 & 35.3 & 7.4 & 231.9 & 146.6 & 40.9 & 9.3 \\
\hline 39 & 7.89 & 187 & 669 & 334 & 169 & 26.1 & 25.0 & 98.1 & 5.6 & 195.3 & 64.6 & 50.7 & 13.7 \\
\hline 40 & 7.84 & 193 & 540 & 270 & 224 & 56.8 & 19.8 & 45.2 & 6.5 & 286.8 & 49.5 & 21.6 & 11.6 \\
\hline 41 & 7.89 & 185 & 340 & 170 & 171 & 59.5 & 5.3 & 16.4 & 1.8 & 146.4 & 9.5 & 39.9 & 15.2 \\
\hline 42 & 7.64 & 201 & 814 & 407 & 368 & 102.5 & 26.7 & 43.7 & 4.8 & 106.8 & 154.5 & 119.7 & 17.4 \\
\hline 43 & 7.93 & 193 & 266 & 133 & 134 & 36.5 & 10.2 & 10.0 & 3.0 & 109.8 & 24.8 & 19.4 & 16.7 \\
\hline 45 & 7.55 & 208 & 793 & 397 & 428 & 100.3 & 42.6 & 33.3 & 4.2 & 347.8 & 93.0 & 37.8 & 16.6 \\
\hline 46 & 7.73 & 199 & 628 & 314 & 210 & 38.1 & 27.5 & 71.0 & 4.0 & 207.5 & 76.0 & 51.4 & 17.4 \\
\hline 47 & 7.64 & 223 & 72 & 36 & 315 & 51.4 & 44.9 & 59.8 & 16.2 & 205.9 & 122.1 & 79.0 & 36.8 \\
\hline 48 & 7.73 & 202 & 433 & 217 & 206 & 44.3 & 23.0 & 24.4 & 3.5 & 183.1 & 49.8 & 25.1 & 12.0 \\
\hline 49 & 7.81 & 199 & 270 & 135 & 107 & 35.7 & 4.3 & 21.0 & 2.7 & 85.4 & 29.9 & 21.6 & 17.3 \\
\hline 50 & 7.62 & 208 & 578 & 289 & 243 & 66.2 & 18.6 & 42.6 & 3.9 & 231.9 & 48.8 & 44.0 & 17.2 \\
\hline 51 & 7.81 & 202 & 311 & 155 & 163 & 40.2 & 15.0 & 10.2 & 2.5 & 97.6 & 53.0 & 13.6 & 13.5 \\
\hline 55 & 7.46 & 230 & 770 & 385 & 362 & 124.1 & 12.4 & 18.2 & 2.3 & 238.0 & 39.9 & 86.7 & 13.7 \\
\hline 56 & 7.55 & 222 & 457 & 229 & 233 & 69.8 & 14.1 & 12.0 & 1.2 & 112.9 & 36.2 & 61.9 & 15.6 \\
\hline 59 & 7.81 & 223 & 300 & 150 & 178 & 29.9 & 24.7 & 3.3 & 0.7 & 137.3 & 5.5 & 17.7 & 21.1 \\
\hline 60 & 7.72 & 233 & 211 & 106 & 107 & 35.7 & 4.4 & 5.7 & 1.5 & 42.7 & 26.9 & 27.7 & 11.9 \\
\hline
\end{tabular}


average selenium concentration of the water samples was $0.154 \mu \mathrm{g} / \mathrm{L}$ (maximum $0.898 \mu \mathrm{g} / \mathrm{L}$ ). The average fluoride concentration was $0.44 \mathrm{mg} / \mathrm{L}$ (maximum $7.24 \mathrm{mg} / \mathrm{L}$ ). However, the average fluoride concentration of the hot-spring water samples was $4.62 \mathrm{mg} / \mathrm{L}$, higher than that of the other samples. The fluoride concentration in Shuanghu county was also high, with an average concentration of about $2.31 \mathrm{mg} / \mathrm{L}$ (Tables 5 and 6).

\subsection{Test results of toxic elements}

The average arsenic concentration in water samples (except the hot-spring water samples) in Tibet was $8.66 \mu \mathrm{g} / \mathrm{L}$. The arsenic concentration in northern Tibet (Damxung, Shuanghu, Gerze, Geji and Seng-ge Kambab) was higher than that in other areas of Tibet, with an average value of about $113.23 \mu \mathrm{g} / \mathrm{L}, 11$ times higher than the Standards for Drinking Water Quality (MH, 2006; $10 \mu \mathrm{g} / \mathrm{L}$ ) (Tables 7 and 8). The water in these areas is therefore not suitable for drinking.

Table 5 Concentrations of trace elements in surface water samples from Tibet

\begin{tabular}{|c|c|c|c|c|c|c|c|c|c|c|c|c|c|}
\hline $\begin{array}{l}\text { Sample } \\
\text { number }\end{array}$ & $\begin{array}{c}\mathrm{Li} \\
(\mu \mathrm{g} / \mathrm{L})\end{array}$ & $\begin{array}{c}\mathrm{Sr} \\
(\mathrm{mg} / \mathrm{L})\end{array}$ & $\begin{array}{c}\text { B } \\
(\mathrm{mg} / \mathrm{L})\end{array}$ & $\begin{array}{c}\mathrm{Zn} \\
(\mu \mathrm{g} / \mathrm{L})\end{array}$ & $\begin{array}{c}\mathrm{Se} \\
(\mu \mathrm{g} / \mathrm{L})\end{array}$ & $\begin{array}{c}\mathrm{F} \\
(\mathrm{mg} / \mathrm{L})\end{array}$ & $\begin{array}{c}\mathrm{U} \\
(\mu \mathrm{g} / \mathrm{L})\end{array}$ & $\begin{array}{c}\mathrm{Rb} \\
(\mu \mathrm{g} / \mathrm{L})\end{array}$ & $\begin{array}{c}\mathrm{Ba} \\
(\mu \mathrm{g} / \mathrm{L})\end{array}$ & $\begin{array}{c}\text { Co } \\
(\mu \mathrm{g} / \mathrm{L})\end{array}$ & $\begin{array}{c}\mathrm{Cs} \\
(\mu \mathrm{g} / \mathrm{L})\end{array}$ & $\begin{array}{c}\mathrm{Ga} \\
(\mu \mathrm{g} / \mathrm{L})\end{array}$ & $\begin{array}{c}\mathrm{V} \\
(\mu \mathrm{g} / \mathrm{L})\end{array}$ \\
\hline 1 & 0.25 & 0.10 & 0.01 & 1.48 & 0.00 & 0.02 & 0.20 & 0.36 & 4.43 & 0.04 & 0.00 & 0.20 & 0.08 \\
\hline 2 & 0.87 & 0.09 & 0.01 & 0.36 & 0.20 & 0.04 & 0.15 & 0.05 & 0.42 & 0.06 & 0.01 & 0.01 & -0.11 \\
\hline 5 & 0.16 & 0.07 & 0.01 & 7.77 & 0.00 & 0.04 & 0.24 & 0.28 & 1.56 & 0.03 & 0.46 & 0.08 & 0.77 \\
\hline 6 & 2.29 & 0.05 & 0.09 & 3.48 & 0.00 & 0.04 & 0.03 & 0.62 & 3.16 & 0.06 & 0.10 & 0.17 & 0.11 \\
\hline 7 & 0.33 & 0.04 & 0.01 & 2.16 & 0.00 & 0.04 & 0.02 & 0.31 & 1.86 & 0.04 & 0.03 & 0.10 & -0.12 \\
\hline 8 & 2.20 & 0.05 & 0.10 & 1.97 & 0.03 & 0.04 & 0.04 & 0.51 & 2.65 & 0.07 & 0.06 & 0.13 & 0.04 \\
\hline 9 & 0.38 & 0.04 & 0.01 & 1.12 & 0.00 & 0.04 & 0.02 & 0.37 & 1.92 & 0.05 & 0.04 & 0.11 & -0.06 \\
\hline 12 & 0.92 & 0.06 & 0.01 & 1.86 & 0.00 & 0.06 & 0.71 & 0.77 & 3.26 & 0.03 & 0.03 & 0.17 & -0.06 \\
\hline 14 & 12.6 & 0.14 & 0.12 & 0.57 & 0.01 & 0.25 & 5.57 & 0.65 & 11.97 & 0.07 & 0.04 & 0.54 & -0.07 \\
\hline 15 & 10.2 & 0.49 & 0.09 & 1.17 & 0.08 & 0.36 & 3.28 & 1.99 & 7.77 & 0.07 & 0.83 & 0.38 & -0.07 \\
\hline 21 & 8.23 & 0.09 & 0.11 & 3.06 & 0.02 & 0.17 & 0.18 & 1.42 & 5.74 & 0.10 & 0.17 & 0.36 & 0.31 \\
\hline 26 & 42.5 & 0.37 & 0.25 & 0.92 & 0.07 & 0.16 & 1.13 & 5.10 & 50.22 & 0.08 & 2.22 & 2.24 & -0.30 \\
\hline 27 & 14.4 & 0.26 & 0.22 & 0.55 & 0.01 & 0.16 & 3.37 & 0.57 & 25.26 & 0.10 & 0.03 & 1.10 & -0.16 \\
\hline 35 & 140 & 3.05 & 1.50 & 2.91 & 0.52 & 0.58 & 10.85 & 0.42 & 13.08 & 0.10 & 0.18 & 0.56 & 0.27 \\
\hline 36 & 18.3 & 0.30 & 0.18 & 57.33 & 0.25 & 0.15 & 0.93 & 1.84 & 29.23 & 0.03 & 0.69 & 1.28 & 0.28 \\
\hline 38 & 8.87 & 0.25 & 0.24 & 1.97 & 0.11 & 0.13 & 1.58 & 0.50 & 7.19 & 0.05 & 0.10 & 0.32 & -0.23 \\
\hline 44 & 162 & 0.24 & 0.52 & 1.72 & 0.18 & 0.18 & 1.20 & 6.96 & 16.13 & 0.05 & 18.83 & 0.69 & -0.36 \\
\hline 52 & 22.3 & 0.69 & 0.34 & 4.18 & 0.85 & 0.04 & 1.35 & 7.76 & 12.15 & 0.06 & 12.05 & 0.50 & -0.43 \\
\hline 53 & 2.48 & 0.18 & 0.07 & 5.80 & 0.13 & 0.15 & 0.31 & 0.66 & 13.27 & 0.09 & 0.28 & 0.60 & -0.43 \\
\hline 54 & 2.59 & 0.08 & 0.14 & 3.70 & 0.19 & 0.07 & 0.08 & 0.78 & 5.95 & 0.03 & 0.07 & 0.24 & -0.36 \\
\hline 57 & 2.67 & 0.12 & 0.10 & 2.46 & 0.22 & 0.24 & 0.52 & 0.17 & 1.29 & 0.04 & 0.03 & 0.05 & -0.41 \\
\hline 58 & 7.3 & 0.29 & 0.21 & 1.45 & 0.45 & 0.14 & 0.95 & 0.27 & 5.07 & 0.05 & 0.09 & 0.21 & -0.09 \\
\hline
\end{tabular}


Table 6 Concentrations of trace elements in groundwater samples from Tibet

\begin{tabular}{|c|c|c|c|c|c|c|c|c|c|c|c|c|c|}
\hline $\begin{array}{l}\text { Sample } \\
\text { number }\end{array}$ & $\begin{array}{c}\mathrm{Li} \\
(\mu \mathrm{g} / \mathrm{L})\end{array}$ & $\begin{array}{c}\mathrm{Sr} \\
(\mathrm{mg} / \mathrm{L})\end{array}$ & $\begin{array}{c}\mathrm{B} \\
(\mathrm{mg} / \mathrm{L})\end{array}$ & $\begin{array}{c}\mathrm{Zn} \\
(\mu \mathrm{g} / \mathrm{L})\end{array}$ & $\begin{array}{c}\mathrm{Se} \\
(\mu \mathrm{g} / \mathrm{L})\end{array}$ & $\begin{array}{c}\mathrm{F} \\
(\mathrm{mg} / \mathrm{L})\end{array}$ & $\begin{array}{c}\mathrm{U} \\
(\mu \mathrm{g} / \mathrm{L})\end{array}$ & $\begin{array}{c}\mathrm{Rb} \\
(\mu \mathrm{g} / \mathrm{L})\end{array}$ & $\begin{array}{c}\mathrm{Ba} \\
(\mu \mathrm{g} / \mathrm{L})\end{array}$ & $\begin{array}{c}\text { Co } \\
(\mu \mathrm{g} / \mathrm{L})\end{array}$ & $\begin{array}{c}\mathrm{Cs} \\
(\mu \mathrm{g} / \mathrm{L})\end{array}$ & $\begin{array}{c}\mathrm{Ga} \\
(\mu \mathrm{g} / \mathrm{L})\end{array}$ & $\begin{array}{c}\mathrm{V} \\
(\mu \mathrm{g} / \mathrm{L})\end{array}$ \\
\hline 3 & 48.9 & 0.15 & 0.18 & 55.80 & 0.00 & 0.09 & 2.39 & 6.08 & 6.49 & 0.05 & 5.45 & 0.30 & 0.15 \\
\hline 4 & 50.3 & 0.15 & 0.20 & 7.68 & 0.00 & 0.12 & 2.41 & 6.49 & 11.98 & 0.06 & 9.06 & 0.58 & 0.09 \\
\hline 10 & 2.97 & 0.15 & 0.13 & 1.16 & 0.00 & 0.04 & 0.30 & 0.09 & 33.48 & 0.07 & 0.02 & 1.66 & -0.08 \\
\hline 11 & 1.15 & 0.09 & 0.02 & 21.54 & 0.00 & 0.06 & 0.21 & 0.07 & 25.74 & 0.04 & 0.16 & 1.33 & -0.16 \\
\hline 13 & 105 & 1.03 & 0.37 & 7.14 & 0.04 & 0.38 & 5.54 & 4.49 & 87.75 & 0.58 & 0.99 & 4.22 & 0.38 \\
\hline 16 & 12.7 & 0.27 & 0.15 & 3.40 & 0.00 & 0.08 & 1.95 & 0.57 & 64.37 & 0.31 & 0.03 & 3.10 & 0.21 \\
\hline 17 & 95.3 & 1.40 & 0.49 & 1.47 & 0.05 & 0.48 & 5.83 & 0.88 & 24.06 & 0.41 & 0.02 & 1.07 & 0.32 \\
\hline 18 & 54.4 & 0.31 & 0.31 & 0.99 & 0.03 & 0.26 & 2.15 & 5.88 & 21.20 & 0.07 & 2.06 & 0.98 & 0.32 \\
\hline 19 & 60.6 & 0.29 & 0.18 & 2.06 & 0.01 & 0.43 & 1.49 & 4.53 & 18.29 & 0.10 & 0.03 & 0.85 & -0.27 \\
\hline 20 & 85.0 & 0.27 & 0.18 & 10.43 & 0.00 & 0.27 & 0.24 & 5.68 & 53.84 & 0.65 & 0.02 & 2.54 & -0.26 \\
\hline 22 & 79.8 & 0.80 & 0.36 & 4.40 & 0.05 & 0.22 & 4.09 & 9.31 & 71.36 & 0.36 & 0.64 & 3.12 & 2.19 \\
\hline 23 & 121.77 & 0.28 & 0.41 & 3.24 & 0.00 & 0.33 & 0.93 & 2.87 & 8.80 & 0.06 & 0.03 & 0.38 & -0.04 \\
\hline 24 & 24.6 & 0.45 & 0.32 & 2.00 & 0.03 & 0.10 & 2.93 & 1.01 & 60.78 & 0.34 & 0.01 & 2.70 & 0.20 \\
\hline 25 & 39.9 & 0.88 & 0.22 & 4.79 & 0.08 & 0.19 & 1.65 & 2.14 & 73.71 & 0.09 & 0.04 & 3.24 & -0.12 \\
\hline 28 & 10.4 & 0.25 & 0.21 & 24.22 & 0.07 & 0.20 & 1.48 & 0.38 & 42.85 & 0.06 & 0.01 & 1.92 & 0.34 \\
\hline 29 & 37.4 & 0.63 & 0.22 & 22.15 & 0.05 & 0.19 & 22.77 & 0.46 & 41.98 & 0.23 & 0.01 & 1.81 & -0.06 \\
\hline 30 & 35.7 & 0.26 & 0.31 & 2.90 & 0.05 & 0.33 & 1.44 & 1.19 & 26.70 & 0.05 & 0.04 & 1.18 & 0.13 \\
\hline 31 & 745 & 1.64 & 2.92 & 18.34 & 0.04 & 3.05 & 0.42 & 82.40 & 94.15 & 0.28 & 85.13 & 4.50 & -0.58 \\
\hline 32 & 2658 & 1.96 & 8.61 & 1.94 & 0.13 & 7.24 & 0.06 & 190.64 & 75.57 & 0.10 & 428.09 & 3.33 & 0.10 \\
\hline 33 & 1495 & 1.77 & 5.35 & 1.80 & 0.06 & 3.56 & 0.31 & 125.09 & 102.19 & 0.05 & 144.69 & 4.69 & -0.22 \\
\hline 34 & 295 & 0.86 & 1.18 & 2.47 & 0.15 & 1.34 & 1.45 & 33.29 & 104.00 & 0.11 & 48.34 & 4.75 & 0.81 \\
\hline 37 & 29.9 & 0.47 & 0.60 & 6.76 & 0.75 & 0.27 & 13.46 & 2.93 & 33.63 & 0.10 & 15.38 & 1.44 & -0.09 \\
\hline 39 & 96.5 & 0.51 & 1.62 & 6.16 & 0.22 & 0.32 & 4.74 & 1.46 & 43.89 & 0.13 & 0.07 & 1.96 & 2.06 \\
\hline 40 & 142 & 0.67 & 1.05 & 1.76 & 0.16 & 0.33 & 3.75 & 18.55 & 104.26 & 0.09 & 7.65 & 4.64 & 3.42 \\
\hline 41 & 18.98 & 0.23 & 0.43 & 2.12 & 0.08 & 0.14 & 1.93 & 0.35 & 43.80 & 0.08 & 0.07 & 1.97 & 0.13 \\
\hline 42 & 64.7 & 0.71 & 1.06 & 3.44 & 0.10 & 0.26 & 11.61 & 0.57 & 28.43 & 0.20 & 0.08 & 1.23 & 0.13 \\
\hline 43 & 27.7 & 0.19 & 0.77 & 2.91 & 0.09 & 0.24 & 1.30 & 1.19 & 11.28 & 0.05 & 0.11 & 0.49 & -0.23 \\
\hline 45 & 55.0 & 0.79 & 0.60 & 2.59 & 0.24 & 0.39 & 2.71 & 3.17 & 25.84 & 0.09 & 1.66 & 1.11 & -0.13 \\
\hline 46 & 49.5 & 0.55 & 1.11 & 0.99 & 0.49 & 0.33 & 2.29 & 1.09 & 24.86 & 0.05 & 0.07 & 1.09 & 0.55 \\
\hline 47 & 458 & 0.45 & 2.11 & 428.69 & 0.53 & 1.20 & 9.08 & 51.88 & 23.02 & 0.08 & 119.46 & 0.98 & 1.84 \\
\hline 48 & 116 & 0.79 & 0.96 & 68.11 & 0.57 & 0.19 & 1.86 & 14.48 & 36.96 & 0.05 & 70.10 & 1.64 & 0.28 \\
\hline 49 & 33.0 & 0.16 & 1.39 & 15.97 & 0.14 & 0.14 & 1.26 & 5.20 & 12.03 & 0.05 & 0.99 & 0.52 & 0.43 \\
\hline 50 & 159 & 0.41 & 2.32 & 6.95 & 0.09 & 0.14 & 3.41 & 6.22 & 30.70 & 0.17 & 6.99 & 1.33 & 0.34 \\
\hline 51 & 30.3 & 0.41 & 0.58 & 5.94 & 0.24 & 0.11 & 1.72 & 2.06 & 39.20 & 0.04 & 0.77 & 1.72 & -0.01 \\
\hline 55 & 14.2 & 0.46 & 0.07 & 3.80 & 0.11 & 0.04 & 2.27 & 0.25 & 9.52 & 0.15 & 0.04 & 0.39 & -0.23 \\
\hline 56 & 7.49 & 0.33 & 0.10 & 3.35 & 0.90 & 0.06 & 0.86 & 0.68 & 17.18 & 0.10 & 0.78 & 0.76 & -0.25 \\
\hline 59 & 3.12 & 0.12 & 0.09 & 1.19 & 0.18 & 0.07 & 0.43 & 0.43 & 6.84 & 0.04 & 0.45 & 0.28 & -0.29 \\
\hline 60 & 31.9 & 0.13 & 0.15 & 24.38 & 0.18 & 0.11 & 1.14 & 3.75 & 6.17 & 0.04 & 5.46 & 0.26 & -0.47 \\
\hline
\end{tabular}


Table 7 Concentrations of toxic elements in surface water samples from Tibet

\begin{tabular}{|c|c|c|c|c|c|c|c|c|c|c|c|}
\hline $\begin{array}{l}\text { Sample } \\
\text { number }\end{array}$ & $\begin{array}{c}\mathrm{Al} \\
(\mu \mathrm{g} / \mathrm{L})\end{array}$ & $\begin{array}{c}\text { As } \\
(\mu \mathrm{g} / \mathrm{L})\end{array}$ & $\begin{array}{c}\mathrm{Be} \\
(\mu \mathrm{g} / \mathrm{L})\end{array}$ & $\underset{(\mu \mathrm{g} / \mathrm{L})}{\mathrm{Cr}}$ & $\begin{array}{c}\mathrm{Cu} \\
(\mu \mathrm{g} / \mathrm{L})\end{array}$ & $\begin{array}{c}\mathrm{Fe} \\
(\mu \mathrm{g} / \mathrm{L})\end{array}$ & $\begin{array}{c}\mathrm{Mn} \\
(\mu \mathrm{g} / \mathrm{L})\end{array}$ & $\begin{array}{c}\text { Mo } \\
(\mu \mathrm{g} / \mathrm{L})\end{array}$ & $\begin{array}{c}\mathrm{Ni} \\
(\mu \mathrm{g} / \mathrm{L})\end{array}$ & $\begin{array}{c}\mathrm{Pb} \\
(\mu \mathrm{g} / \mathrm{L})\end{array}$ & $\begin{array}{c}\mathrm{Se} \\
(\mu \mathrm{g} / \mathrm{L})\end{array}$ \\
\hline 1 & 3.26 & 1.10 & 0.05 & 6.77 & 0.31 & 76.00 & 0.09 & 1.56 & 0.71 & 0.00 & 0.00 \\
\hline 2 & 0.37 & 2.05 & 0.04 & 10.71 & 0.21 & 106.38 & 0.06 & 0.72 & 0.93 & 0.01 & 0.20 \\
\hline 5 & 0.59 & 89.38 & 0.06 & 9.79 & -0.05 & 66.10 & 0.04 & 0.21 & 0.57 & 0.00 & 0.00 \\
\hline 6 & 87.74 & 0.90 & 0.03 & 4.90 & 0.64 & 68.68 & 0.65 & 0.23 & 0.55 & 0.06 & 0.00 \\
\hline 7 & 16.93 & 0.30 & 0.03 & 2.96 & 0.54 & 36.31 & 0.13 & 0.23 & 0.53 & 0.02 & 0.00 \\
\hline 8 & 60.61 & 0.76 & 0.09 & 3.10 & 0.57 & 60.99 & 2.44 & 0.24 & 0.54 & 0.05 & 0.03 \\
\hline 9 & 26.73 & 0.31 & 0.04 & 2.58 & 0.51 & 43.60 & 0.41 & 0.25 & 0.44 & 0.04 & 0.00 \\
\hline 12 & 3.57 & 10.59 & 0.01 & 4.97 & 0.09 & 51.28 & 0.11 & 0.61 & 0.50 & 0.00 & 0.00 \\
\hline 14 & 1.34 & 0.68 & 0.05 & 5.65 & 0.94 & 97.90 & 1.45 & 0.71 & 2.08 & 0.02 & 0.01 \\
\hline 15 & 0.80 & 1.74 & 0.03 & 4.83 & 0.16 & 121.11 & 0.36 & 1.58 & 1.19 & 0.04 & 0.08 \\
\hline 21 & 385.72 & 0.86 & -0.02 & 6.87 & 0.78 & 251.87 & 2.75 & 0.27 & 1.23 & 0.25 & 0.02 \\
\hline 26 & 4.52 & 1.51 & -0.06 & 2.42 & 0.26 & 134.14 & 0.04 & 0.33 & 1.54 & 0.01 & 0.07 \\
\hline 27 & 2.00 & 1.36 & -0.04 & 2.01 & 0.38 & 167.27 & 0.34 & 1.06 & 2.02 & 0.02 & 0.01 \\
\hline 35 & 0.98 & 1.63 & -0.04 & 2.39 & 1.52 & 140.65 & 0.09 & 2.80 & 1.95 & 0.01 & 0.52 \\
\hline 36 & 0.30 & 2.22 & -0.04 & 6.03 & 0.86 & 50.03 & 0.11 & 0.80 & 0.51 & 0.05 & 0.25 \\
\hline 38 & 2.20 & 2.96 & -0.01 & 1.18 & 0.18 & 87.86 & 0.05 & 0.58 & 0.91 & 0.00 & 0.11 \\
\hline 44 & 0.99 & 3.44 & -0.07 & 1.09 & -0.02 & 109.53 & 0.16 & 0.64 & 1.02 & 0.01 & 0.18 \\
\hline 52 & 1.45 & 1.81 & 0.00 & 1.91 & -0.03 & 146.57 & 0.13 & 0.34 & 1.86 & 0.01 & 0.85 \\
\hline 53 & 84.26 & 0.73 & -0.02 & 0.67 & 0.37 & 75.09 & 6.62 & 0.52 & 1.06 & 0.05 & 0.13 \\
\hline 54 & 42.07 & 6.97 & 0.02 & 0.62 & 0.13 & 44.11 & 0.55 & 1.51 & 0.54 & 0.07 & 0.19 \\
\hline 57 & 1.08 & 1.20 & -0.05 & 1.08 & -0.07 & 75.46 & 0.27 & 0.88 & 0.73 & 0.01 & 0.22 \\
\hline 58 & 0.70 & 2.00 & -0.08 & 8.40 & 0.08 & 91.71 & 0.11 & 0.44 & 0.87 & 0.01 & 0.45 \\
\hline
\end{tabular}

\section{Discussion}

\subsection{Hydrochemical characteristics}

The water samples collected (except for sample numbers 20 and 30) were weakly alkaline (Tables 3 and 4) and the $\mathrm{pH}$ of these water samples met both the Chinese national standard and the international standard (WHO, 2004; MH, 2006) (Table 9). The TDS and TH in the natural waters of Tibet also met both the Chinese national standard and the international standard (WHO, 2004; MH, 2006). Some of the water samples also met the national Drink Natural Mineral Water Standard (GAQS, 2008) (samples collected from Kema village, Luoma town, Nagchu county; Marm town, Gerze county; and Baga town, Burang county; Table 9).

\subsection{Toxic elements}

Arsenic poisoning is a common endemic disease. The main symptom of arsenic poisoning is 
Table 8 Concentrations of toxic elements in groundwater samples from Tibet

\begin{tabular}{|c|c|c|c|c|c|c|c|c|c|c|c|}
\hline $\begin{array}{l}\text { Sample } \\
\text { number }\end{array}$ & $\begin{array}{c}\mathrm{Al} \\
(\mu \mathrm{g} / \mathrm{L})\end{array}$ & $\begin{array}{c}\text { As } \\
(\mu \mathrm{g} / \mathrm{L})\end{array}$ & $\begin{array}{c}\mathrm{Be} \\
(\mu \mathrm{g} / \mathrm{L})\end{array}$ & $\begin{array}{c}\mathrm{Cr} \\
(\mu \mathrm{g} / \mathrm{L})\end{array}$ & $\begin{array}{c}\mathrm{Cu} \\
(\mu \mathrm{g} / \mathrm{L})\end{array}$ & $\begin{array}{c}\mathrm{Fe} \\
(\mu \mathrm{g} / \mathrm{L})\end{array}$ & $\begin{array}{c}\mathrm{Mn} \\
(\mu \mathrm{g} / \mathrm{L})\end{array}$ & $\begin{array}{c}\text { Mo } \\
(\mu \mathrm{g} / \mathrm{L})\end{array}$ & $\begin{array}{c}\mathrm{Ni} \\
(\mu \mathrm{g} / \mathrm{L})\end{array}$ & $\begin{array}{c}\mathrm{Pb} \\
(\mu \mathrm{g} / \mathrm{L})\end{array}$ & $\begin{array}{c}\mathrm{Se} \\
(\mu \mathrm{g} / \mathrm{L})\end{array}$ \\
\hline 3 & 0.19 & 1.96 & -0.02 & 10.35 & -0.04 & 98.39 & 0.07 & 0.96 & 0.77 & 0.01 & 0.00 \\
\hline 4 & 0.46 & 3.10 & -0.04 & 10.63 & 0.02 & 100.00 & 0.02 & 0.80 & 0.82 & 0.00 & 0.00 \\
\hline 10 & 99.64 & 0.48 & 0.06 & 9.66 & 0.23 & 96.70 & 0.39 & 0.10 & 0.86 & 0.01 & 0.00 \\
\hline 11 & 0.55 & 1.06 & -0.04 & 8.46 & -0.01 & 59.88 & 0.83 & 0.08 & 0.53 & 0.03 & 0.00 \\
\hline 13 & 0.95 & 0.38 & 0.00 & 13.97 & 1.55 & 344.86 & 242.76 & 0.59 & 5.75 & 0.09 & 0.04 \\
\hline 16 & 1.10 & 0.54 & 0.04 & 18.13 & 1.07 & 257.68 & 0.11 & 0.16 & 2.71 & 0.01 & 0.00 \\
\hline 17 & 7.28 & 0.58 & -0.01 & 12.06 & 0.88 & 238.17 & 0.09 & 0.39 & 2.61 & 0.01 & 0.05 \\
\hline 18 & 33.97 & 5.09 & 0.05 & 3.48 & 0.62 & 78.13 & 0.30 & 0.67 & 1.60 & 0.02 & 0.03 \\
\hline 19 & 0.75 & 0.64 & -0.01 & 7.97 & 0.19 & 148.33 & 0.23 & 0.24 & 1.46 & 0.01 & 0.01 \\
\hline 20 & 0.49 & 0.61 & -0.07 & 16.32 & 0.04 & 143.27 & 514.97 & 0.21 & 1.84 & 0.01 & 0.00 \\
\hline 22 & 1.15 & 1.05 & -0.03 & 6.78 & 3.35 & 320.31 & 19.15 & 0.41 & 5.85 & 0.02 & 0.05 \\
\hline 23 & 0.49 & 0.63 & -0.03 & 4.62 & 0.50 & 112.17 & 0.55 & 0.15 & 1.13 & 0.01 & 0.00 \\
\hline 24 & 0.30 & 0.47 & 0.02 & 4.87 & 0.84 & 269.51 & 0.11 & 0.10 & 3.46 & 0.01 & 0.03 \\
\hline 25 & 0.26 & 1.20 & -0.08 & 2.86 & 0.31 & 140.86 & 0.03 & 0.51 & 1.40 & 0.00 & 0.08 \\
\hline 28 & 1.19 & 0.87 & 0.00 & 2.98 & 0.15 & 65.05 & 0.08 & 0.20 & 0.75 & 0.01 & 0.07 \\
\hline 29 & 4.38 & 3.21 & -0.08 & 3.77 & 0.59 & 246.08 & 0.55 & 0.91 & 2.45 & 0.02 & 0.05 \\
\hline 30 & 0.44 & 1.54 & -0.02 & 2.00 & 0.12 & 62.22 & 0.06 & 0.99 & 0.66 & 0.02 & 0.05 \\
\hline 31 & 0.67 & 46.19 & 0.14 & 60.90 & 2.51 & 333.99 & 2.82 & 2.25 & 4.22 & 0.02 & 0.04 \\
\hline 32 & 2.13 & 333.92 & 0.07 & 19.04 & 7.56 & 125.91 & 5.81 & 0.11 & 1.64 & 0.07 & 0.13 \\
\hline 33 & 0.63 & 219.94 & 0.23 & 33.60 & 4.51 & 110.92 & 6.62 & 0.30 & 1.03 & 0.02 & 0.06 \\
\hline 34 & 0.47 & 2.46 & -0.03 & 9.48 & 0.82 & 155.70 & 0.20 & 0.58 & 1.60 & 0.02 & 0.15 \\
\hline 37 & 0.45 & 1.29 & 0.00 & 3.63 & 0.94 & 156.06 & 0.06 & 2.19 & 1.79 & 0.01 & 0.75 \\
\hline 39 & 0.62 & 6.81 & -0.04 & 6.45 & 1.16 & 45.64 & 0.07 & 2.19 & 0.74 & 0.01 & 0.22 \\
\hline 40 & 1.35 & 4.42 & -0.05 & 1.84 & 0.53 & 107.69 & 0.04 & 0.59 & 1.25 & 0.01 & 0.16 \\
\hline 41 & 1.27 & 3.77 & -0.03 & 1.49 & 0.17 & 107.52 & 0.00 & 0.47 & 1.10 & 0.01 & 0.08 \\
\hline 42 & 0.67 & 2.22 & 0.05 & 1.64 & 0.84 & 190.51 & 0.01 & 2.36 & 2.07 & 0.05 & 0.10 \\
\hline 43 & 0.49 & 4.46 & -0.04 & 1.20 & 0.13 & 65.08 & 0.01 & 0.54 & 0.65 & 0.01 & 0.09 \\
\hline 45 & 0.47 & 1.28 & 0.04 & 3.68 & 0.04 & 188.20 & 0.04 & 1.06 & 1.89 & 0.01 & 0.24 \\
\hline 46 & 0.43 & 3.03 & -0.02 & 7.20 & 0.29 & 64.15 & 0.42 & 1.02 & 0.70 & 0.01 & 0.49 \\
\hline 47 & 0.31 & 163.55 & -0.06 & 1.50 & 0.74 & 89.59 & 0.94 & 6.02 & 0.98 & 0.02 & 0.53 \\
\hline 48 & 0.68 & 57.96 & -0.10 & 2.07 & 0.20 & 80.98 & 0.02 & 1.28 & 0.81 & 0.01 & 0.57 \\
\hline 49 & 7.98 & 56.21 & -0.03 & 0.81 & 0.06 & 64.30 & 0.00 & 0.98 & 0.65 & 0.01 & 0.14 \\
\hline 50 & 0.91 & 17.48 & 0.02 & 1.61 & 0.30 & 122.64 & 0.13 & 0.59 & 1.82 & 0.01 & 0.09 \\
\hline 51 & 0.63 & 1.09 & -0.03 & 1.62 & 0.00 & 74.42 & 0.02 & 0.72 & 0.73 & 0.01 & 0.24 \\
\hline 55 & 0.86 & 1.51 & -0.04 & 5.66 & 0.12 & 238.44 & 0.11 & 0.36 & 2.18 & 0.01 & 0.11 \\
\hline 56 & 21.45 & 3.56 & -0.03 & 2.78 & 0.25 & 133.97 & 2.34 & 0.64 & 1.44 & 0.02 & 0.90 \\
\hline 59 & 0.97 & 2.50 & 0.01 & 4.65 & 0.09 & 53.98 & 0.00 & 0.18 & 0.92 & 0.02 & 0.18 \\
\hline 60 & 1.55 & 1.87 & -0.03 & 1.02 & 0.00 & 65.49 & 0.62 & 1.34 & 0.75 & 0.01 & 0.18 \\
\hline
\end{tabular}


Table 9 Major and trace element test standards for water quality

\begin{tabular}{|c|c|c|c|}
\hline Parameter & $\begin{array}{c}\text { Standards for Drinking } \\
\text { Water Quality (MOHC, } \\
\text { 2006) }\end{array}$ & $\begin{array}{c}\text { Guidelines for Drinking Water } \\
\text { Quality (WHO, 2004) }\end{array}$ & $\begin{array}{c}\text { Drink Natural Mineral } \\
\text { Water (AQSIQ, 2008) }\end{array}$ \\
\hline $\mathrm{pH}$ & $6.5-8.5$ & $6.5-9.5$ & - \\
\hline TDS (mg/L) & 1000 & 1000 & $\geq 1000$ \\
\hline TH (mg/L) & 450 & 500 & - \\
\hline $\mathrm{Na}^{+}(\mathrm{mg} / \mathrm{L})$ & 200 & 200 & - \\
\hline $\mathrm{SO}_{4}^{2-}(\mathrm{mg} / \mathrm{L})$ & 250 & 500 & - \\
\hline $\mathrm{Cl}^{-}(\mathrm{mg} / \mathrm{L})$ & 250 & 250 & - \\
\hline $\mathrm{H}_{2} \mathrm{SiO}_{3}(\mathrm{mg} / \mathrm{L})$ & - & - & $\geq 25.0$ \\
\hline $\mathrm{Li}(\mu \mathrm{g} / \mathrm{L})$ & - & - & $\geq 200$ \\
\hline $\mathrm{Sr}(\mathrm{mg} / \mathrm{L})$ & - & - & $\geq 0.2$ \\
\hline $\mathrm{B}(\mathrm{mg} / \mathrm{L})$ & 0.5 & 0.5 & $<5$ \\
\hline $\mathrm{Zn}(\mu \mathrm{g} / \mathrm{L})$ & 1000 & 3000 & $\geq 200$ \\
\hline $\operatorname{Se}(\mu \mathrm{g} / \mathrm{L})$ & 10 & 10 & $\geq 10$ \\
\hline $\mathrm{F}(\mathrm{mg} / \mathrm{L})$ & 1 & 1.5 & $<1.5$ \\
\hline $\mathrm{U}(\mu \mathrm{g} / \mathrm{L})$ & - & 15 & - \\
\hline $\mathrm{Ba}(\mu \mathrm{g} / \mathrm{L})$ & 700 & 700 & $<700$ \\
\hline
\end{tabular}

skin alteration, including dermal hyperkeratosis, verrucous keratosis and skin cancer (Smith, 1992). Some patients also experience gastrointestinal or hepatic dysfunction (Zhao et al., 2002). Studies have shown that the arsenic concentration in water is mainly affected by the underlying lithology (Qing et al., 2007). In Damxung, Shuanghu, Gerze, Geji and Seng-ge Kambab of northern Tibet, the arsenic concentration is high (Tables 7, 8 and 10). As a result, people who live in these regions may be affected by arsenic poisoning through drinking water. Therefore it is essential to carry out further research on endemic arsenic poisoning in these prefectures as well as on the distribution and genesis of water with a high arsenic concentration. The fluoride concentration in Shuanghu county exceeded both the Chinese national standard and the international standard (WHO, 2004; $\mathrm{MH}, 2006$ ). The toxic elements in water from other regions meet both these standards, which means that water in these regions is suitable for drinking.

\subsection{Hydrochemical characteristics}

The ratio of major ions in water can be clearly shown with a Piper plot (Piper, 1944); the percentage of major ions determines the hydrochemical type of water (Chen et al., 2014; Piper, 1944; Shen et al., 2007; Zhu et al., 2011). The main hydrochemical types of water samples in Tibet are as follows: $\mathrm{Ca}-\mathrm{Mg}-\mathrm{HCO}_{3}$ (eight samples); $\mathrm{Ca}-\mathrm{Mg}-\mathrm{HCO}_{3}-\mathrm{SO}_{4}$ (eight samples); $\mathrm{Ca}-\mathrm{HCO}_{3}$ (five samples); $\mathrm{Ca}-\mathrm{HCO}_{3}-\mathrm{Cl}$ (three samples); $\mathrm{Ca}-\mathrm{Na}-\mathrm{Mg}-\mathrm{HCO}_{3}$ (three samples); $\mathrm{Na}-\mathrm{Ca}-\mathrm{HCO}_{3}$ (three samples); $\mathrm{Ca}-\mathrm{Mg}-\mathrm{HCO}_{3}-\mathrm{Cl}$ (two samples); $\mathrm{Mg}-\mathrm{Ca}-\mathrm{HCO}_{3}$ (two samples); $\mathrm{Na}_{-} \mathrm{HCO}_{3}$ (two samples) (Figure 3; Tables 3 and 4). 
Table 10 Harmful element test standards for water quality

\begin{tabular}{cccc}
\hline Element $(\mu \mathrm{g} / \mathrm{L})$ & $\begin{array}{c}\text { Standards for Drinking Water Quality } \\
(\mathrm{MOHC}, 2006)\end{array}$ & $\begin{array}{c}\text { Guidelines for Drink- } \\
\text { ing-Water Quality } \\
(\text { WHO, 2004) }\end{array}$ & $\begin{array}{c}\text { Drink Natural Mineral Water } \\
\text { (AQSIQ, 2008) }\end{array}$ \\
\hline $\mathrm{Ag}$ & 50 & 100 & - \\
$\mathrm{Al}$ & 200 & 200 & $<50$ \\
$\mathrm{As}$ & 10 & 10 & - \\
$\mathrm{Be}$ & 2 & - & $<3$ \\
$\mathrm{Cd}$ & 5 & 3 & $<50$ \\
$\mathrm{Cr}$ & 50 & 50 & $<1000$ \\
$\mathrm{Cu}$ & 1000 & 2000 & - \\
$\mathrm{Fe}$ & 300 & 300 & $<1$ \\
$\mathrm{Hg}$ & 1 & 1 & $<400$ \\
$\mathrm{Mn}$ & 100 & 400 & - \\
$\mathrm{Mo}$ & 70 & 70 & $<20$ \\
$\mathrm{Ni}$ & 20 & 20 & $<10$ \\
$\mathrm{~Pb}$ & 10 & 10 & $<0$ \\
$\mathrm{Sl}$ & 0.1 & 10 & $<$ \\
\hline
\end{tabular}

The predominant cation and anion in the water samples from Tibet were $\mathrm{Ca}^{2+}$ and $\mathrm{HCO}_{3}^{-}$, respectively. The major cations in water were $\mathrm{Ca}^{2+}>\mathrm{Na}^{+}>\mathrm{Mg}^{2+}>\mathrm{K}^{+}$and the major anions were $\mathrm{HCO}_{3}{ }^{-}>\mathrm{SO}_{4}{ }^{2-}>\mathrm{Cl}^{-}$(Figure 4). From south to north, the main cation in water changed from $\mathrm{Ca}^{2+}$ to $\mathrm{Na}^{+}$, whereas the main anions in water changed from $\mathrm{HCO}_{3}^{-}$to $\mathrm{Cl}^{-}$and $\mathrm{SO}_{4}{ }^{2-}$. The surface runoff and groundwater in Tibet are recharged mainly by ice/snow melt water and rain.

The main water type in northern Tibet, in the Yarlung Zangbo river catchment, the Lhasa river catchment, the Nianchu river catchment, the Nyang river catchment and the internal flow lake basin area is pore water from loose rocks. Bedrock fissure water is mainly distributed from north of the Himalayas to south of the Changtse Mountains. Karst water is mainly distrib-

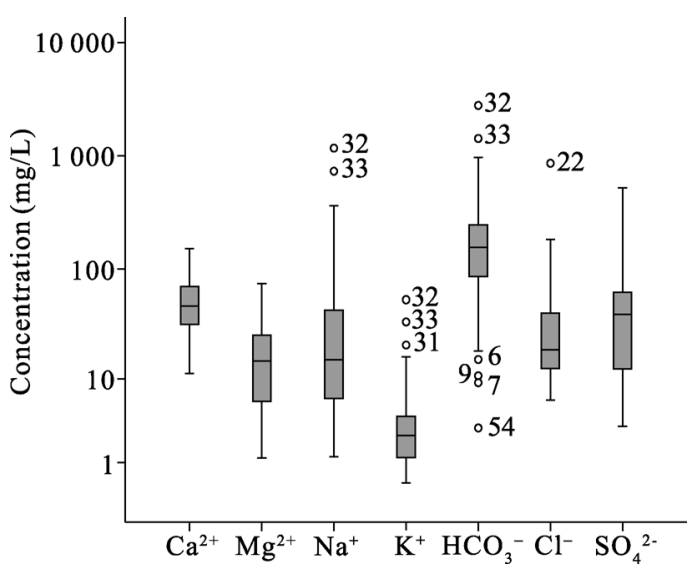

Figure 3 Box and whisker plots showing the variation of major ion concentrations in water samples from Tibet

\subsection{Preliminary discussion on the causes of variation in Tibetan water samples}

The boomerang envelope model developed by Gibbs (1970) describes three types of water: (1) water from evaporation/crystallization; (2) water dominated by rock type; and (3) water 
from atmospheric precipitation. The chemical composition of surface water in Tibet is mainly controlled by rock weathering (Figure 5).

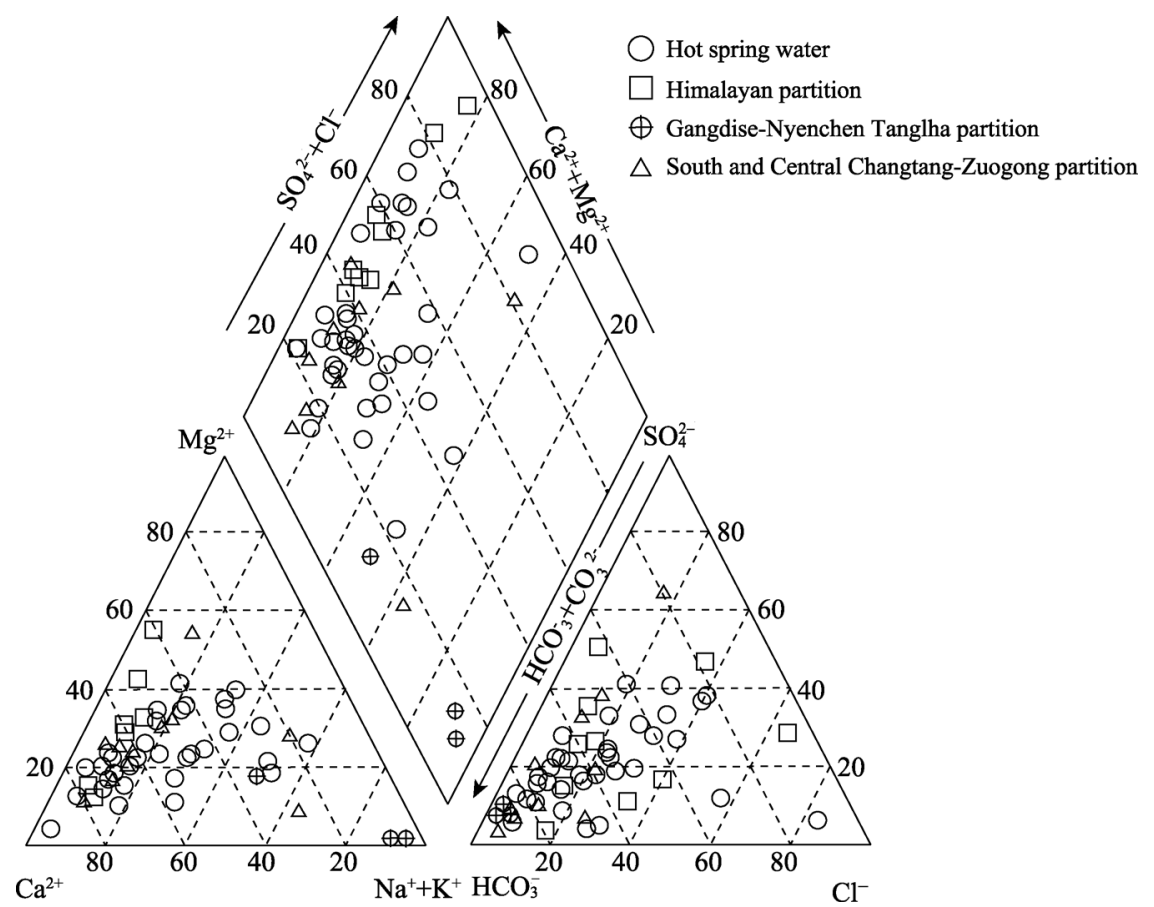

Figure 4 Piper diagram showing the major concentrations of cations and anions in waters of Tibet by geological setting
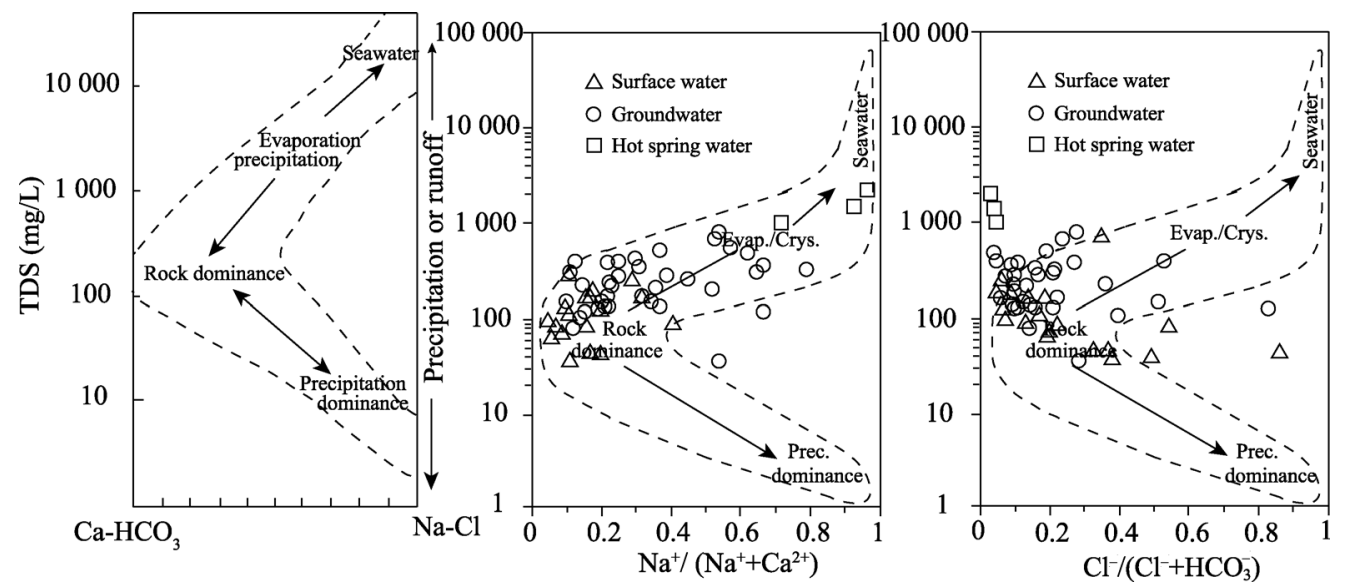

Figure 5 Plots of the major ions within the Gibbs boomerang envelope for waters in Tibet

The control of the chemical composition of surface water in Tibet by rock weathering is consistent with results from elsewhere in the world, including the Yangtze River, the Amazon River and the Ganges (Gibbs, 1970). The results for groundwater in the Gibbs boomerang envelope plot are relatively fragmented, which suggests that the chemical composition of groundwater is diverse and complicated. As a result of the high temperatures, the concentrations of elements in the hot-spring waters are high and water samples are close to the 
seawater type in the Gibbs boomerang envelope.

\subsection{Regional comparison}

In order to explore the differences in the hydrochemical characteristics of different regions, we compared the average values for water samples collected from the Tibetan region with the average values for water samples collected from Southern Xinjiang, the Tongtian River in Qinghai, the Qinghai Lake Basin, Huanglong (Yellow Dragon), Maoxian county and Zamtang county of Sichuan, Yarlung Zangbo River between Lhasa-Nyingchi and Shegyla Mountain (Table 11).

Table 11 Hydrochemical characteristics in different regions of western China

\begin{tabular}{|c|c|c|c|c|c|c|c|c|c|}
\hline & Tibet & $\begin{array}{l}\text { Southern } \\
\text { Xinjiang }\end{array}$ & $\begin{array}{l}\text { Tongtian } \\
\text { River }\end{array}$ & $\begin{array}{c}\text { Qinghai Lake } \\
\text { Basin }\end{array}$ & $\begin{array}{l}\text { Huan- } \\
\text { glong }\end{array}$ & $\begin{array}{l}\text { Maoxian } \\
\text { county }\end{array}$ & $\begin{array}{c}\text { Zamtang } \\
\text { county }\end{array}$ & $\begin{array}{l}\text { Yarlung Zangbo } \\
\text { River between } \\
\text { Lhasa-Nyingchi }\end{array}$ & $\begin{array}{l}\text { Shegyla } \\
\text { Mountain }\end{array}$ \\
\hline $\begin{array}{l}\text { Number of } \\
\text { samples }\end{array}$ & 57 & 154 & 9 & 75 & 9 & 63 & 423 & 62 & 9 \\
\hline $\mathrm{pH}$ & 7.55 & 7.64 & 8.09 & 8.09 & 6.59 & 7.7 & 7.43 & 7.74 & 7.46 \\
\hline $\mathrm{K}^{+}$ & 3.09 & 17.87 & 6.77 & 1.87 & 0.4 & 2.36 & 14.25 & 3.16 & 0.14 \\
\hline $\mathrm{Na}^{+}$ & 34.93 & 336.97 & 118.02 & 36.42 & 3.14 & 19.12 & & 4.89 & 0.03 \\
\hline $\mathrm{Ca}^{2+}$ & 55.25 & 90.1 & 51.77 & 37.38 & 253.78 & 70.36 & 33.77 & 29.74 & 1.6 \\
\hline $\mathrm{Mg}^{2+}$ & 18.69 & 73.78 & 18.82 & 16.04 & 20.94 & 28.38 & 10.4 & 5.56 & 3.82 \\
\hline $\mathrm{Cl}^{-}$ & 47.67 & 509.41 & 179.88 & 47 & 0.73 & 7.37 & 3.9 & 3.87 & 7.41 \\
\hline $\mathrm{SO}_{4}{ }^{2-}$ & 54.68 & 429.08 & 83.34 & 39.35 & 23.2 & 119.6 & 8.05 & 25.83 & 62.52 \\
\hline $\mathrm{HCO}_{3}^{-}$ & $\begin{array}{c}169.6 \\
7\end{array}$ & 260.28 & 170.63 & 183.37 & 777.44 & 226.27 & 171.21 & 90.28 & 33.9 \\
\hline Reference & $\begin{array}{l}\text { This } \\
\text { study }\end{array}$ & $\begin{array}{c}\text { Liu et al. (2014), } \\
\text { Pang et al. } \\
\text { (2010), Zhang } \\
\text { et al. (1995) }\end{array}$ & $\begin{array}{l}\text { Su et al. } \\
\text { (1987) }\end{array}$ & $\begin{array}{l}\text { Xu et al. } \\
(2010)\end{array}$ & $\begin{array}{l}\text { Wang } \\
\text { et al. } \\
(2009)\end{array}$ & $\begin{array}{c}\mathrm{Du} \\
(2011)\end{array}$ & $\begin{array}{l}\text { Cao } \\
(2011)\end{array}$ & Liu (2011) & $\begin{array}{c}\text { Ren et al. } \\
\text { (2002) }\end{array}$ \\
\hline
\end{tabular}

The hydrochemical types of the Southern Xinjiang, Qinghai Tongtian River, Lake Qinghai catchment, Sichuan Yellow Dragon, Sichuan Maoxian county, Sichuan Zamtang county, Yarlung Zangbo River between Lhasa and Nyingchi and Shegyla Mountain are: $\mathrm{Na}-\mathrm{Mg}-\mathrm{Cl}-\mathrm{SO}_{4}, \mathrm{Na}-\mathrm{Ca}-\mathrm{Cl}-\mathrm{HCO}_{3}, \mathrm{Ca}-\mathrm{Na}-\mathrm{Mg}-\mathrm{HCO}_{3}-\mathrm{Cl}, \mathrm{Ca}-\mathrm{HCO}_{3}, \mathrm{Ca}-\mathrm{Mg}-\mathrm{HCO}_{3}-\mathrm{SO}_{4}$, $\mathrm{Ca}-\mathrm{Mg}-\mathrm{HCO}_{3}, \mathrm{Ca}-\mathrm{Mg}-\mathrm{HCO}_{3}-\mathrm{SO}_{4}$ and $\mathrm{Mg}-\mathrm{SO}_{4}-\mathrm{HCO}_{3}$, respectively. It can be inferred that different locations lead to different hydrochemical types (Table 11 and Figure 6).

\section{Conclusions}

The data obtained in this study are representative of the natural hydrochemical characteristics in Tibet as a result of the limited human activities in this region. The water quality in most regions of Tibet is good and meets both the Chinese national standard and the international standard (WHO, 2004; MH, 2006). Some of the water samples also met the national Drink Natural Mineral Water Standard (GAQS, Inspection and Quarantine of the People's Republic of China, 2008). The arsenic and fluoride concentrations in Damxung, Shuanghu, 


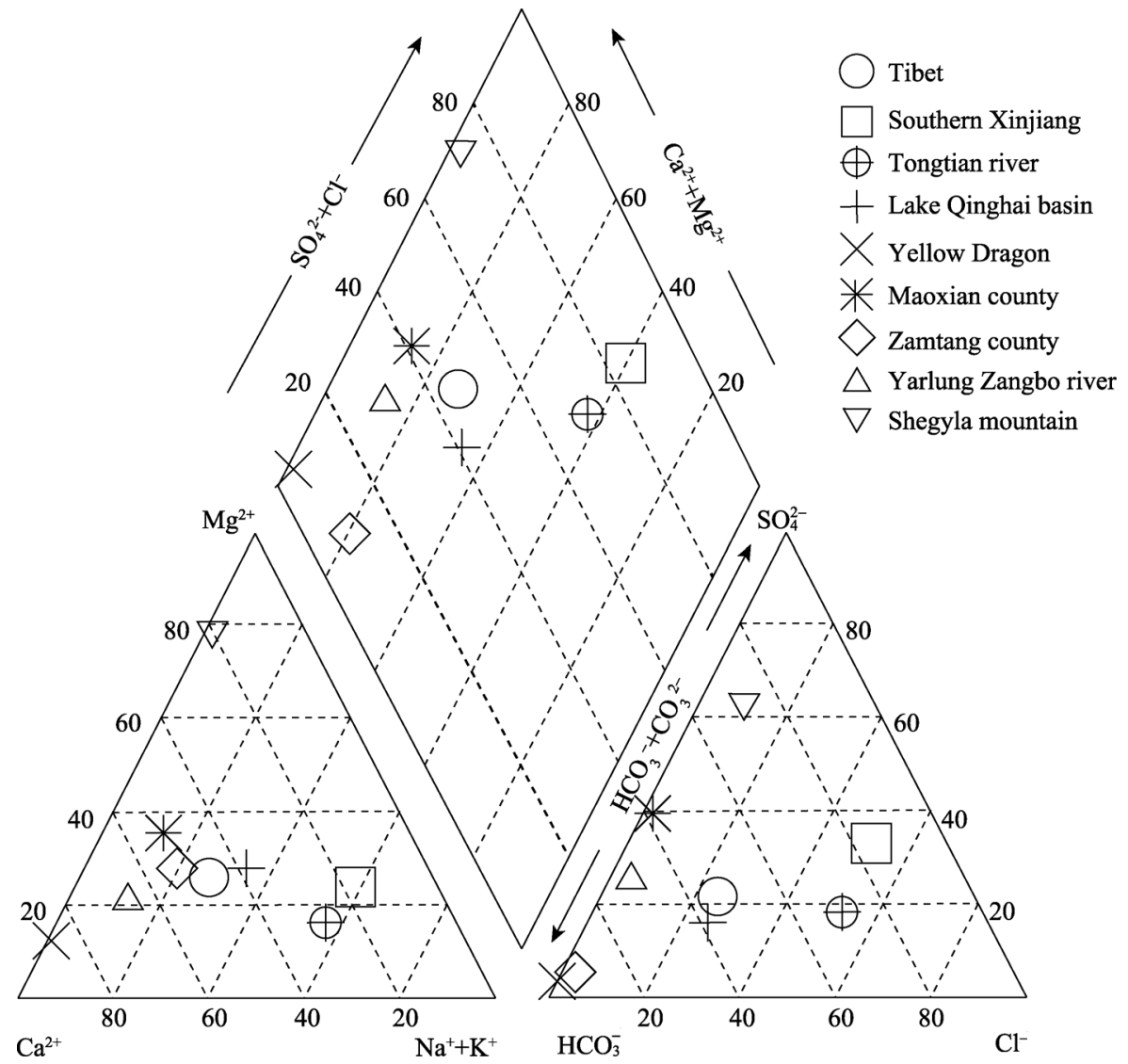

Figure 6 Piper diagram showing major ion compositions of natural water in different regions of western China

Gerze, Geji and Seng-ge Kambab of northern Tibet are higher than those specified in the Chinese national standard and the international standard (MH, 2006; WHO, 2004).

The results of this study can be summarized as follows.

(1) The $\mathrm{pH}$ value of water samples ranges from 6.75 to 8.21 and most of the water samples are weakly alkaline.

(2) The mean value of TDS in water samples is $225.54 \mathrm{mg} / \mathrm{L}$, except for the hot-spring water samples. Therefore the majority of water in Tibet is suitable for drinking.

(3) The arsenic concentration in water samples from Ali prefecture and the fluoride concentration in water samples from Shuanghu exceed both the Chinese national standard and the international standard (WHO, 2004; MH, 2006). Further studies are needed on fluorosis and endemic arsenic poisoning resulting from drinking water.

(4) The dominant ions in water from Tibet are $\mathrm{Ca}^{2+}$ and $\mathrm{HCO}_{3}^{-}$. The main hydrochemical types of Tibetan water are $\mathrm{Ca}-\mathrm{HCO}_{3}, \mathrm{Ca}-\mathrm{Mg}-\mathrm{HCO}_{3}$ and $\mathrm{Ca}-\mathrm{Mg}-\mathrm{HCO}_{3}-\mathrm{SO}_{4}$. From south to north, the main cation in water changes from $\mathrm{Ca}^{2+}$ to $\mathrm{Na}^{+}$, whereas the main anions in water change from $\mathrm{HCO}_{3}^{-}$to $\mathrm{Cl}^{-}$and $\mathrm{SO}_{4}^{2-}$.

(5) River water and ice/snow melt water are dominated by the rock type and the formation of groundwater is affected by many factors. The element concentrations in hot-spring water are high and are similar to seawater. 


\section{References}

Bianduo, Bianbaciren, Laba et al., 2010. The response of water level of Selin Co to climate change during 1975-2008. Acta Geographica Sinica, 65(3): 313-319. (in Chinese)

Buduo, Jiang D S, Ren J Y et al., 2011. Preliminary study on the arsenic water of fisheries in Lhasa Region. Journal of Tibet University, 26(1): 15-24. (in Chinese)

Cao N, 2011. An analysis of the characteristics of geological environment in the Kashin-Beck disease (KBD) area in the Rangtang county [D]. Chengdu: Chengdu University of Technology. (in Chinese)

Chen L, Wang G C, Hu F S et al., 2014. Groundwater hydrochemistry and isotope geochemistry in the Turpan Basin, northwestern China. Journal of Arid Land, 6(4): 378-388.

Du J T, 2011. Hydrogeochemical research of Kashin-Beck disease [D]. Chengdu: Chengdu University of Technology. (in Chinese)

General Administration of Quality Supervision, Inspection and Quarantine of the People's Republic of China (GAQS), 2008. GB 8537-2008. Drinking Natural Mineral Water. (in Chinese)

Gibbs R J, 1970. Mechanisms controlling world water chemistry. Science, 170: 1088-1090.

Hirokazu H, Yoshiki W, Satoshi O, 1985. Silver chloride pre-treatment for the direct potentiometric determination of chloride in stream waters using a solid-state chloride ion-selective electrode. Analyst, 110(9): 1087-1090.

Ji Q G, Wang B Q, 2007. The data processing and application of groundwater chemical composition. Land and Resources of Southern China, 2007(1): 31-33. (in Chinese)

Li H J, Zhang N, Lin X T, 2010. Spatio-temporal characteristics of Yarlung Zangbo River in Tibet. Journal of Henan Normal University, 38(2): 126-130. (in Chinese)

Liu C, Gesangcuomu, 2012. Direct determination 22 kinds of trace elements of lake water in Tibet by ICP-MS. Tibet's Science \& Technology, (2): 27-31. (in Chinese)

Liu X Y, Shi Y, 2013. Tibet Shannan rural drinking water safety project in 2012 water quality monitoring results. Journal of Tibet Medicine, 35(3): 54-55. (in Chinese)

Liu Y L, Luo K L, Lin X X et al., 2014. Regional distribution of longevity population and chemical characteristics of natural water in Xinjiang, China. Science of the Total Environment, 473/474: 54-62.

Liu Zhao, 2011. The characterization of hydrochemical and isotopic in the natural water of the Yarlung Tsangpo Lhasa-Nyingchi [D]. Chengdu: Chengdu University of Technology. (in Chinese)

Luo D, Huang C Y, Yin T, 2010. Assessment of water quality and water security on centralized drinking water sources in towns of Tibet. Water Conservancy Science and Technology and Economy, 16(4): 420-422. (in Chinese)

Ma L F, Deng X Z, 2002. China Geological Map Explanation. Beijing: Geological Press. (in Chinese)

Ministry of Environment Protection of the People's Republic of China, 2002. Methods for Chemical Analysis of Water and Waste Water. 4th ed. Beijing: China Environmental Science Press. (in Chinese)

Ministry of Health (MH) of the People's Republic of China, 1985. GB5750 85. Standards for Drinking Water Test. (in Chinese)

Ministry of Health (MH) of the People's Republic of China, 2006. GB 5749-2006. Standards for Drinking Water Quality. (in Chinese)

Ministry of Water Resources (MWR) of the People's Republic of China, 2009. China's Water Resource Report in 2007. (in Chinese)

Nie L X, 2011. Drinking water microorganism index analysis of 6 counties in Tibet rural area. Journal of Tibet Medicine, 32(1): 56-57. (in Chinese)

Pang Z H, Huang T M, Chen Y N, 2010. Diminished groundwater recharge and circulation relative to degrading riparian vegetation in the middle Tatim River, Xinjiang Uygur, Western China. Hydrol. Process, 24: 147-59.

Piper M A, 1944. A graphic procedure in the geochemical interpretation of water-analyses. Transactions, American Geophysical Union, 25: 914-928.

Qing Z Y, Jing Z, Ying W et al., 2007. Hydrochemical processes controlling arsenic and selenium in the Changjiang River (Yangtze River) system. Science of the Total Environment, 377(1): 93-104. 
Ren Q S, Wang J S, Zhang B et al., 2002. Different forms of water quality analysis in southeastern Tibetan fir forest. Journal of Northeast Forestry University, 30(2): 52-54. (in Chinese)

Shen Y L, Zhou M W, 2011. The environmental geological assessment in Tibet. Sichuan Acta Geologica Sinica, 31: 89-92. (in Chinese)

Shen Z L, Zhu W H, 1993. Hydrogeochemical Basis. Beijing: Geological Publishing House, 83-91. (in Chinese)

Smith A H, Hopenhayn-Rich C, Bates M N et al., 1992. Cancer risks from arsenic in drinking water. Environ. Health Perspectives, 97: 259-267.

Su C J, Tang B X, 1987. The hydrochemical characteristics of Tongtian River. Mountain Research, 5(3): $143-146$. (in Chinese)

Wang H J, Liu Z H, Zheng C, 2009. Hydrochemical variations of Huanglong spring and the stream in Huanglong ravine, Sichuan province. Geochimica, 38(3): 307-314. (in Chinese)

Wang J B, Peng P, Ma Q F et al., 2013. Investigation of water depth, water quality and modern sedimentation rate in Mapam Yumco and La'ang Co, Tibet. Journal of Lake Sciences, 25(4): 609-616. (in Chinese)

Warren V H, 1989. Geology, trace elements and health. Social Science \& Medicine, 29(8): 923-926.

Webster J G, Brown K L, Vincent W F, 1994. Geochemical processes affecting ice-snow melting water chemistry and the formation of saline ponds in the Victoria Valley and Bull Pass region, Antarctica. Hydrobiologia, 281(3): 171-186.

World Health Organization (WHO), 2004. Guidelines for Drinking Water Quality. Recommendation 1, 3rd ed. World Health Organization, Geneva, 306-308.

Xu H, Hou Z H, An Z S et al., 2010. Major ion chemistry of waters in lake Qinghai catchments, NE Qinghai-Tibet Plateau, China. Quaternary International, 2010, 212: 35-43.

Yu C Z, Fu E H, 2010. Determination of chloride in water by ion-selective electrode method. Chemical Analysis and Meterage, 19(1): 40-42. (in Chinese)

Zhang N, Li H J, Wen Z Z et al., 2009. Spatio-temporal characteristics of Niyang River in Tibet. Journal of Henan Normal University, 37(6): 79-82. (in Chinese)

Zhang J, Takahashi K, Wushiki H et al., 1995. Water geochemistry of the rivers around the Taklimakan desert (NW China): Crustal weathering and evaporation processes in arid land. Chemical Geology, 119: $225-37$.

Zhang X Y, Li X J, Dawa et al., 2013. Drinking water hygiene monitoring and analysis in 2011, Tibet Nyingchi. Journal of Tibet Medicine, 34(1): 62-64. (in Chinese)

Zhao S L, Wang L F, Liang J H, 2002. The damage and remove measure of arsenic in drinking water. Modern Preventive Medicine, 2002(5): 651-652. (in Chinese)

Zhao W, Liu D H, Zhang H P et al., 2002. Investigation of water quality on individual supply well in Tibet servicemen outstation. Journal of PLA Preventive Medicine, 20(6): 427-428. (in Chinese)

Zheng B M, Li S Z, Li S S et al., 2007. The analysis and significance of water quality in certain department troops adopt the provide for oneself centered type water supply dwell in Tibet Naqu district. Journal of Henan Preventive Medicine, 18(5): 332-334. (in Chinese)

Zheng B M, Li S Z, Ma K J et al., 2007. The investigation and analysis of the spring and well water of certain part halt in Tibet Biru country. Journal of Henan Preventive Medicine, 18(4): 245-246. (in Chinese)

Zheng B M, Li S Z, Zhou X B, 2008. The detection of 11 bathhouses's bathwater quality at certain part and habitat in Tibet Naqu. Journal of Henan Preventive Medicine, 19(3): 168-171. (in Chinese)

Zhu B Q, Yang X P, Rioual P et al., 2011. Hydrogeochemistry of three watersheds (the Erlqis, Zhungarer and Yili) in northern Xinjiang, NW China. Applied Geochemistry, 26(8): 1535-1548. 